\title{
BOUNDARY VALUES OF AN ANALYTIC FUNCTION AND THE TCHEBYCHEFF METHOD OF APPROXIMATION*
}

BY

\author{
J. L. WALSH
}

1. Introduction. If the functions $f(z), f_{n}(z), n=1,2, \cdots$, are analytic on and within the unit circle $C$ of the $z$-plane, the relation

$$
\lim _{n \rightarrow \infty}\left[\max \left|f(z)-f_{n}(z)\right|, z \text { on } C\right]=0
$$

implies the relation

$$
\lim _{n \rightarrow \infty} f_{n}(z)=f(z)
$$

uniformly for $z$ on and within $C$.

There are, however, cases of approximation formally similar to thiswhich consist, namely, of approximation on the boundary $C$ of a region $B$ by functions analytic on $B+C$, to a function defined and continuous on $C-$ where approximation with an arbitrarily small error is not possible. For instance, the function $f(z)$ to be approximated may be the boundary values of an analytic function with singularities in $B$, or the approximating functions $f_{n}(z)$ may all be required to take on prescribed values different from those of $f(z)$ at definite points interior to $B$; in neither of these cases is (1.1) possible. Natural questions to be raised in these new cases are: (1) What is the minimum value $M$ (or greatest lower bound) of

$$
\max \left|f(z)-f_{n}(z)\right|, z \text { on } C,
$$

for all functions $f_{n}(z)$ analytic interior to $C$ and taking on the prescribed values if any? (2) Does a function $f_{0}(z)$, taking on the prescribed values if any, exist such that

$$
\max \left|f(z)-f_{0}(z)\right|=M, \text { for } z \text { on } C \text { ? }
$$

(3) If we have

$$
\lim _{n \rightarrow \infty}\left[\max \left|f(z)-f_{n}(z)\right|, z \text { on } C\right]=M,
$$

where the functions $f_{n}(z)$ are analytic interior to $C$ and take on the prescribed

* Presented to the Society, March 30,1929; received by the editors December 16, 1929, and April 7,1930 . An abstract of this paper was published in the Proceedings of the National Academy of Sciences, vol. 15 (1929), pp. 799-802. 
values if any, what can be said of the convergence of the sequence $\left\{f_{n}(z)\right\}$ ? (4) In particular, if we consider as a special sequence the Tchebycheff polynomials for approximation to $f(z)$ on $C$, what can be said of the convergence?

There are two theorems of primary interest which in the simplest cases answer these questions:

THEOREM A. Let $B$ be an arbitrary simply connected region with the boundary $C$, and let the function $f(z)$ be analytic on $C$, meromorphic on $B+C$. Then there exists a function $f_{0}(z)$ analytic interior to $B$ such that*

$$
\underset{\substack{z \rightarrow C \\ z \text { in } B}}{\bar{b}}\left|f(z)-f_{0}(z)\right|=M
$$

is less than

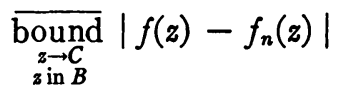

or any other function $f_{n}(z)$ analytic interior to $B$. The expression $\left|f(z)-f_{0}(z)\right|$ approaches the same limit $M$ no matter how $z$ in $B$ approaches $C$. If the functions $f_{n}(z), n=1,2, \cdots$, are analytic interior to $B$, then

$$
\lim _{n \rightarrow \infty}\left[\overline{\substack{\text { bound } \\ z \rightarrow C \\ \sin B}}\left|f(z)-f_{n}(z)\right|\right]=M
$$

implies

$$
\lim _{n \rightarrow \infty} f_{n}(z)=f_{0}(z)
$$

for $z$ in $B$, uniformly for $z$ on an arbitrary closed point set interior to $B$.

If the region $B$ is limited and if its boundary $C$ is also the boundary of an infinite region, the Tchebycheff polynomial $\omega_{n}(z)$ of degree $n$ for approximation to $f(z)$ on $C$ is defined as the polynomial of degree $n$ such that

$$
\max \left|f(z)-\omega_{n}(z)\right|, z \text { on } C,
$$

is less than the corresponding expression formed for any other polynomial of degree $n$; the Tchebycheff polynomial $\omega_{n}(z)$ exists and is unique. It is then true if $f(z)$ has no singularities other than in $B$ and in the infinite region of which $C$ is the boundary, that

$$
\lim _{n \rightarrow \infty}\left[\max \left|f(z)-\omega_{n}(z)\right|, z \text { on } C\right]=M,
$$

from which it follows that

$$
\lim _{n \rightarrow \infty} \omega_{n}(z)=f_{0}(z)
$$

for $z$ in $B$, uniformly on any closed point set interior to $B$.

* The notation here is intended to imply that we allow $z$ in $B$ to approach $C$ in any way whatever and take the least upper bound of all corresponding limits of $\left|f(z)-f_{0}(z)\right|$. 
If $B$ is the interior of a limited analytic Jordan curve $C$, we denote by $C_{R}$ the curve which is the locus $|\phi(z)|=R>1$, where $w=\phi(z)$ is a function which maps the exterior of $C$ onto the exterior of the unit circle $|w|=1$ so that the points at infinity correspond to each other. In this case we have (1.2) valid not merely for $z$ in $B$ but also for $z$ interior to the largest curve $C_{R}$ which contains in its interior no singularity of $f_{0}(z)$-such a curve $C_{R}$ exists and is exterior to $C$-and the convergence in (1.2) is uniform on an arbitrary closed point set interior to this $C_{R}$.

In the second theorem we shall be concerned with approximation on the boundary $C$ of a region $B$ by functions which satisfy certain auxiliary conditions at a finite number of preassigned points $P$ interior to $B$. These conditions are, at each point $P$, prescription of the value of the function together with the values of its first $k_{P}$ derivatives.

THEOREM B. Let $B$ be an arbitrary simply connected region with the boundary $C$, and let the function $F(z)$ be analytic on $B+C$. Then there exists a function $F_{0}(z)$ analytic interior to $B$ satisfying arbitrary given auxiliary conditions, such that

$$
M=\overline{\text { bound }}\left|\vec{F}(z)-F_{0}(z)\right|, z \text { in } B,
$$

is less than

$$
\overline{\text { bound }}\left|F(z)-F_{n}(z)\right|, z \text { in } B \text {, }
$$

for any other function $F_{n}(z)$ analytic interior to $B$ and satisfying the same auxiliary conditions. The expression $\left|F(z)-F_{0}(z)\right|$ approaches the same limit $M$ no matter how $z$ in $B$ approaches $C$. If the functions $F_{n}(z), n=1,2, \cdots$, are analytic interior to $B$ and satisfy the given auxiliary conditions, then

implies

$$
\lim _{n \rightarrow \infty}\left[\overline{\text { bound }}\left|F(z)-F_{n}(z)\right|, z \text { in } B\right]=M
$$

$$
\lim _{n \rightarrow \infty} F_{n}(z)=F_{0}(z)
$$

for $z$ in $B$, uniformly for $z$ on an arbitrary closed point set interior to $B$.

If the region $B$ is limited and if its boundary $C$ is also the boundary of an infinite region, the Tchebycheff polynomial $\pi_{n}(z)$ of degree $n$ for approximation to $F(z)$ on $C$ with the given auxiliary conditions is the polynomial of degree $n$ which satisfies the auxiliary conditions such that

$$
\max \left|F(z)-\pi_{n}(z)\right|, z \text { in } B,
$$

is less than the corresponding expression formed for any other polynomial of degree $n$ and satisfying the auxiliary conditions; this Tchebycheff polynomial 
$\pi_{n}(z)$ exists for $n$ sufficiently large and is unique. It is true if $F(z)$ has no singularities other than in the infinite region bounded by $C$, that

$$
\lim _{n \rightarrow \infty}\left[\max \left|F(z)-\pi_{n}(z)\right|, z \text { in } B\right]=M,
$$

from which it follows that

$$
\lim _{n \rightarrow \infty} \pi_{n}(z)=F_{0}(z)
$$

for $z$ in $B$, uniformly on any closed point set interior to $B$.

If $B$ is the interior of a limited analytic Jordan curve $C$ and if $C_{R}$ has the same generic meaning as before, we have (1.3) valid not merely for $z$ in $B$ but also for $z$ interior to the largest curve $C_{R}$ which contains in its interior no singularity of $F_{0}(z)$-such a curve exists and is exterior to $C$-and the convergence in (1.3) is uniform on an arbitrary closed point set interior to this $C_{R}$.

These two theorems and generalizations are to be proved by methods and results due to Carathéodory and Fejér, Gronwall, Schur, R. Nevanlinna, Carathéodory, and Tonelli. Some of the results of Theorem $\mathrm{B}$ have recently been obtained by Julia, for special auxiliary conditions of the form $F_{n}(0)=0$, $F_{n}^{\prime}(0)=1$. This case is connected with the conformal mapping of the region $B$ onto a circle, as is the case of Theorem A for which $f(z)$ is of the form $1 / z$.

F. Riesz has recently considered* an integral measure of approximation and states without giving any details that his methods can be used in studying approximation to rational functions by analytic functions. Those methods are not closely related to the ones that we use here.

The remainder of the present paper is devoted largely to proofs of the various parts of Theorems $A$ and $B$, although some of our results are more detailed than is suggested by the statements just given, and others, particularly in \$9, are considerably more general than Theorems A and B. In fact Theorems 14 and 15 together make a unit which includes both Theorems $A$ and $B$ as special cases.

2. Existence and uniqueness of minimizing function for a circle. The first part of Theorem B is now to be proved for the special case of a circle:

THEOREM 1. Of the class of all functions, each of which is analytic for $|z|<1$ and each of which at $k$ given points $P$ interior to $C:|z|=1$ takes on preassigned values (the same for all functions of the class) for the function and its first $k_{P}$ derivatives, a unique function $F(z)$ has the least upper bound to its absolute value for $|z|<1$. This function $F(z)$ is a rational function of $z$ and of constant modulus $M$ on $C$.

\footnotetext{
* Acta Mathematica, vol. 42 (1920), pp. 145-171.
} 
This theorem was established by Cartheodory and Fejér* for the case of a single point $P$, new methods were introduced by Gronwall $\dagger$ and Schur, $\ddagger$ the theorem was proved by Pick $\S$ for arbitrary $k$ but $k_{P}=0$, and the general theorem is to be established by methods and results due to R. Nevanlinna.\| Nevanlinna has derived necessary and sufficient conditions that a function satisfying auxiliary conditions of the kind we are considering be of modulus less than unity interior to $C$.

We indicate the proof $\mathbb{T}$ of Theorem 1 . There exists at least one function analytic for $|z| \leqq 1$ which satisfies the auxiliary conditions at the points $P$ and which has a limited modulus for $|z| \leqq 1$; indeed, a polynomial can easily be written down which has this property. Hence there is a finite lower limit $M$ to the upper bounds of the moduli for $|z|<1$ of all functions $F(z)$ analytic for $|z|<1$ which satisfy the auxiliary conditions. There exists a function $F(z)$ analytic for $|z|<1$ and satisfying the auxiliary conditions, whose modulus for $|z|<1$ has the upper bound $M$. For there exist functions $F_{n}(z)$ analytic for $|z|<1$ and satisfying the auxiliary conditions, such that

$$
\overline{\text { bound }}\left|F_{n}(z)\right| \leqq M+\frac{1}{n}, \text { for }|z|<1, n=1,2, \cdots \text {. }
$$

These functions $F_{n}(z)$ form a normal family in $|z|<1$, and from them can be extracted a subsequence which converges for $|z|<1$, uniformly for $|z| \leqq \rho<1$. Let the limit of this subsequence be denoted by $F(z)$. Then the function $F(z)$ also satisfies the auxiliary conditions, by the uniformity of the convergence, and we have in each point of $|z|<1$

$$
|F(z)| \leqq M
$$

by the conditions on the $F_{n}(z)$.

The uniqueness of this function $F(z)$ analytic and of modulus not greater than $M$ for $|z|<1$ and satisfying the auxiliary conditions, follows readily from the results of Nevanlinna. We consider the new function** $F(z) / M$, with the obvious corresponding modifications to be made in the auxiliary conditions in the given points. If the function $F(z)$ is not unique, we have, in the

* Palermo Rendiconti, vol. 32 (1911), pp. 218-239.

$\dagger$ Annals of Mathematics, (2), vol. 14 (1912-13), pp. 72-80; vol. 16 (1914-15), pp. 77-81.

$\ddagger$ Crelle’s Journal, vol. 147 (1916), pp. 205-232; vol. 148 (1917), pp. 122-145.

$\S$ Mathematische Annalen, vol. 77 (1915), pp. 7-23; Annales Academiae Scientiarum Fennicae, (A), vol. 15 (1921).

\| Annales Academiae Scientiarum Fennicae, (A), vol. 13 (1920), pp. 1-71.

I Compare Gronwall, loc. cit.

** The case that the prescribed values of function and derivatives in the auxiliary conditions are all zero is trivial and henceforth excluded. In any other case we have $M \neq 0$. 
notation of Nevanlinna (loc. cit., pp. 15, 36),

$$
\left|\phi_{n}\right|<1 \text {, }
$$

where our function $F(z) / M$ plays the rôle of Nevanlinna's function $z(x)$, and where $n+1$ is the sum of the $k_{P}$. We can then choose a particular $z^{(n)}(x)$ identically equal to $\phi_{n}$, a constant of absolute value less than unity. Then there exists a function $z(x)$ defined by Nevanlinna's recurrent equation (22) [if $k_{P}=0$ and by the corresponding equation if $k_{P}>0$ ], analytic for $|z| \leqq 1$ and satisfying the new auxiliary conditions. For this function it is true and follows directly from Nevanlinna's equation (22) and from our (2.1) that for $|z| \leqq 1$ we have

$$
\left|z^{(n-1)}(x)\right|<\rho_{n-1}<1, \quad\left|z^{(n-2)}(x)\right|<\rho_{n-2}<1, \cdots,|z(x)|<\rho<1 .
$$

A special case of these inequalities, namely, $\left|\phi_{i}\right|<1, i=0,1, \cdots, n-1$, is indeed necessary for the existence of the function $z(x)$. Thus we have, for some function $F(z)$ satisfying our original auxiliary conditions,

$$
\left|\frac{F(z)}{M}\right|<\rho<1, \text { for }|z| \leqq 1,
$$

which contradicts our definition of $M$.

We remark, and this follows from Nevanlinna's results, that our function $F(z)$ is of degree less than $n+1$, the sum of the $k_{P}$.

The effective determination of the number $M$ of Theorem 1 in any given case does not seem to be a simple matter. It will be noticed, however, that Nevanlinna's equation (pp. 15, 36)

$$
\left|\phi_{n}\left(x_{0}, z_{0} ; \cdots ; x_{n}, z_{n}\right)\right|=1,
$$

where $n$ has the same significance as before, is an algebraic* equation which has $M$ for a root. Here the quantities $z_{i}$, etc., are not precisely the values which occur in the auxiliary conditions of Theorem 1, but are the quotients of those values by $M$. The condition on $M$ represented by (2.2) is necessary (see also below) that $M$ be the number of Theorem 1, because necessary for the uniqueness of Nevanlinna's function $z(x)$, but is not sufficient. The sufficiency arises only if Nevanlinna's inequalities

$$
\left|\phi_{0}\right|<1, \cdots,\left|\phi_{n-1}\left(x_{0}, z_{0} ; \cdots ; x_{n-1}, z_{n-1}\right)\right|<1,
$$

where the $z_{i}$ are again the quantities of Theorem 1 divided by $M$, are also

* Equation (2.2) is not in its present form an algebraic equation, but becomes such if the various absolute values are suitably written and the corresponding equation simplified. There may be ambiguity, however, for the sign of a term expressed as an absolute value (such as $\left|M^{2}-\alpha\right|$ ) may depend on the value of $M$, which is itself determined by the equation. 
satisfied. These inequalities are not implied by (2.2), as simple examples show.* But equation (2.2) has a unique positive root for which inequalities (2.3) are satisfied. For we shall reach a contradiction by assuming that there exist two such roots $M_{1}$ and $M_{2}$, with $M_{1}<M_{2}$. There exists (Nevanlinna, pp. 15,36$)$ a unique function $F_{1}(z)$ which takes on the prescribed auxiliary values and is of modulus not greater than $M_{1}$ in $C$ and also a unique function $F_{2}(z)$ which takes on the prescribed auxiliary values and is of modulus not greater than $M_{2}$ in $C$. This contradicts the obvious fact that the polynomial

$$
F_{1}(z)+\epsilon \prod\left(z-\alpha_{i}\right)
$$

where the $\alpha_{i}$ are the points $P$ and appear respectively $k_{P}$ times in the product, also satisfies the auxiliary conditions and for suitable sufficiently small $\epsilon$ is of modulus less than $M_{2}$ and yet not equal to $F_{2}(z)$.

Nevanlinna's condition (2.2), (2.3) is, as a matter of fact, not necessary and sufficient for the uniqueness of $z(x)$, and hence (2.2) is not in its present form a necessary condition on $M$. For it may occur that we have

$$
\begin{gathered}
\left|\phi_{0}\right|<1,\left|\phi_{1}\right|<1, \cdots,\left|\phi_{i-1}\right|<1,\left|\phi_{i}\right|=1, i<n, \\
z_{\nu}^{(i)}\left(x_{0}, z_{0} ; \cdots ; x_{i-1}, z_{i-1} ; x_{\nu}, z_{\nu}\right)=\phi_{i}, \nu=i+1, i+2, \cdots, n,
\end{gathered}
$$

in which case also $z(x)$ exists and is unique. In this case the functions $z^{(i+1)}(x), \cdots, z^{(n)}(x)$ and quantities $\left|\phi_{i+1}\right|, \cdots,\left|\phi_{n}\right|$ take the form $0 / 0$ and are, properly speaking, not defined. But equation (2.2) can be written and transformed so that it is automatically satisfied also if (2.4) holds, and in this sense our previous statement is true, that (2.2) is an algebraic equation of which $M$ is a root.

3. Continuity of the function $F(z)$. We shall prove now, for later application, that the function $F(z)$ (of Theorem 1) varies continuously with the auxiliary conditions. That is to say, if $\epsilon^{\prime}>0$ be given, there exists $\delta$ such that the inequalities

$$
\left|\alpha_{i}-\alpha_{i}^{\prime}\right|<\delta, \quad\left|\gamma_{i}-\gamma_{i}^{\prime}\right|<\delta \quad(i=1,2, \cdots, k)
$$

imply that

$$
\left|F(z)-F^{\prime}(z)\right|<\epsilon^{\prime} \text { for }|z| \leqq 1,
$$

where $F(z)$ is the function of Theorem 1 for the auxiliary conditions

$$
F\left(\alpha_{i}\right)=\gamma_{i} \quad(i=1,2, \cdots, k),
$$

and $F^{\prime}(z)$ is the function of Theorem 1 for the auxiliary conditions

$$
F^{\prime}\left(\alpha_{i}^{\prime}\right)=\gamma_{i}^{\prime} \quad(i=1,2, \cdots, k) .
$$

* For instance, $x_{0}=0, x_{1}=1 / 2, z_{0}=2, z_{1}=5 / 4$. 
Here the values $\alpha_{i}$ and $\gamma_{i}$ are considered fixed, while $\alpha_{i}^{\prime}$ and $\gamma_{i}^{\prime}$ are arbitrary, subject merely to the restrictions (3.1). The number $\delta$ will then depend on $\epsilon^{\prime}$ and on the $\alpha_{i}$ and $\gamma_{i}$, but not on the $\alpha_{i}^{\prime}$ and $\gamma_{i}^{\prime}$. It is reasonable to suppose that $F(z)$ varies continuously with the auxiliary conditions, for $F(z)$ is determined from those auxiliary conditions entirely by algebraic processes. We give, however, a formal proof.

As a preliminary proposition we shall prove that the absolute value $M$ of $F(z)$ for $|z|=1$ varies continuously with the auxiliary conditions. That is, if $\epsilon>0$ be given, there exists $\delta$ such that inequalities (3.1) imply

$$
M-\epsilon<M^{\prime}<M+\epsilon,
$$

where $M^{\prime}$ is the absolute value of $F^{\prime}(z)$ for $|z|=1$. It seems reasonable too that $M$ should be a continuous function of the auxiliary conditions, for $M$ is a root of an algebraic equation whose coefficients depend rationally on those conditions. But this algebraic equation may depend, as we have already suggested, to some extent on the value of $M$ itself, so we prefer to give a detailed proof based on other considerations.

It is possible so to determine $\delta$ that whenever we have

$$
\left|\alpha_{i}-a_{i}\right|<\delta_{1},\left|\gamma_{i}-c_{i}\right|<2 \delta,
$$

where $2 \delta_{1}$ is less than every $\left|\alpha_{i}-\alpha_{j}\right|, i \neq j$, then there exists* a polynomial $p(z)$ which takes on the values $c_{i}-\gamma_{i}$ at the points $a_{i}$ and is such that $|p(z)| \leqq \epsilon$ for $|z| \leqq 1$. The numbers $\alpha_{i}$ and $\gamma_{i}$ are as before considered fixed, determined by the auxiliary conditions (3.3), while the $a_{i}$ and $c_{i}$ are subject only to the restriction of inequalities (3.6). All functions analytic and of absolute value not greater than $M+\epsilon$ for $|z| \leqq 1$ are equicontinuous for $|z| \leqq \rho<1$. We choose $\rho$ greater than every $\left|\alpha_{i}\right|$, and then still further restrict $\delta_{1}$ in (3.6) if necessary so that $\delta_{1}+\left|\alpha_{i}\right|<\rho$ and so that functional values at two arbitrary points in $|z| \leqq \rho$ whose distance apart is less than $\delta_{1}$, of an arbitrary function analytic and of modulus not greater than $M+\epsilon$ in $|z| \leqq 1$, differ by less than $\delta$.

We shall now prove (3.5) under the assumption that the $\delta$ of (3.1) is the smaller of the two quantities $\delta_{1}$ and $\delta$ of (3.6). Let us consider the polynomial $p(z)$ such that

$$
p\left(\alpha_{i}^{\prime}\right)=F^{\prime}\left(\alpha_{i}^{\prime}\right)-F\left(\alpha_{i}\right)+\left[F\left(\alpha_{i}\right)-F\left(\alpha_{i}^{\prime}\right)\right]=\gamma_{i}^{\prime}-\gamma_{i}+\left[F\left(\alpha_{i}\right)-F\left(\alpha_{i}^{\prime}\right)\right],
$$

which is in absolute value less than $2 \delta$. We have $|p(z)| \leqq \epsilon$, for $|z| \leqq 1$, and

* Walsh, these Transactions, vol. 30 (1928), pp. 307-332. See the proof of the Lemma, p. 319. We are using in the present paper essentially a generalization of that Lemma, where the functional values $c_{i}-\gamma_{i}$ of the polynomial concerned are restricted only by the second of inequalities (3.6), and the points $a_{i}$ at which those values are taken on are restricted only by the first of those inequalities. 
hence $|F(z)+p(z)| \leqq M+\epsilon$, for $|z| \leqq 1$, where $F\left(\alpha_{i}^{\prime}\right)+p\left(\alpha_{i}^{\prime}\right)=F^{\prime}\left(\alpha_{i}^{\prime}\right)=\gamma_{i}^{\prime}$. That is, we have exhibited a function $F_{1}(z)=F(z)+p(z)$ analytic for $|z| \leqq 1$ whose functional values are $\gamma_{i}^{\prime}$ for $z=\alpha_{i}^{\prime}$, and whose modulus for $|z| \leqq 1$ is not greater than $M+\epsilon$. Thus we have proved $M^{\prime} \leqq M+\epsilon$.

We determine next a new polynomial $p^{\prime}(z)$ such that

$$
\begin{aligned}
p^{\prime}\left(\alpha_{i}\right) & =F\left(\alpha_{i}\right)-F^{\prime}\left(\alpha_{i}^{\prime}\right)+\left[F^{\prime}\left(\alpha_{i}^{\prime}\right)-F^{\prime}\left(\alpha_{i}\right)\right] \\
& =\gamma_{i}-\gamma_{i}^{\prime}+\left[F^{\prime}\left(\alpha_{i}^{\prime}\right)-F^{\prime}\left(\alpha_{i}\right)\right],
\end{aligned}
$$

which is in absolute value less than $2 \delta$; we have already shown $\left|F^{\prime}(z)\right| \leqq M+\epsilon$ for $|z| \leqq 1$, so the square bracket is in absolute value less than $\delta$. It follows that $\left|p^{\prime}(z)\right| \leqq \epsilon$ for $|z| \leqq 1$, and hence that $\left|F^{\prime}(z)+p^{\prime}(z)\right| \leqq M^{\prime}+\epsilon$ for $|z| \leqq 1$, where $F^{\prime}\left(\alpha_{i}\right)+p^{\prime}\left(\alpha_{i}\right)=F\left(\alpha_{i}\right)=\gamma_{i}$. That is, we have exhibited a function $F_{2}(z)=F^{\prime}(z)+p^{\prime}(z)$ analytic for $|z| \leqq 1$ whose functional values are $\gamma_{i}$ for $z=\alpha_{i}$, and whose absolute value for $|z| \leqq 1$ is not greater than $M^{\prime}+\epsilon$. Thus we have proved $M \leqq M^{\prime}+\epsilon$, and the preliminary proposition (3.5) is established.

To be sure, we have given the proof only for the case $k_{P}=0$, but this is simply a matter of convenience in exposition. The modifications necessary for arbitrary $k_{P}$ involve inequalities of form (3.1) also for the derivatives of $F(z)$ at the points $\alpha_{i}$, but are fairly obvious and hence are left to the reader. This remark applies also below.

We can now easily prove the desired continuity of the function $F(z)$. For the formulas given by Nevanlinna (i.e. his (22)), suitably modified for functions of modulus not greater than $M$ instead of not greater than unity, give us $F(z)$ explicitly in terms of $M$ and of the quantities involved in the auxiliary conditions. First we determine $\phi_{n}$ in terms of these quantities and then express $z(x)$ (modified) in terms of $\phi_{r}$ and these quantities. These formulas for $F(z)$ in terms of all these quantities are indeed valid even if we have Nevanlinna's equation (30) for some $i<n$. In the modified (22) for instance, we have

$$
\frac{z^{(i)}(x)}{M}=\frac{\xi_{i} \frac{z^{(i+1)}(x)}{M}+\frac{\phi_{i}}{M}}{1+\frac{\bar{\phi}_{i} \xi_{i} z^{(i+1)}(x)}{M^{2}}} ;
$$

if $z^{(i+1)}(x)$ approaches the limit $\phi_{i}$, which must occur if we are to have (30) and $\left(30^{\prime}\right)$ fulfilled, then we have $\left|\phi_{i}\right|=M$, and hence we find $\lim z^{(i)}(x)=\phi_{i}$, uniformly throughout the unit circle. The functions $F(z)$ involved are in every case seen by inspection to be continuous functions of $M$ and of the auxiliary conditions, for $z$ on and within $C$, and our theorem is established. 
It may be remarked that a simpler proof of this theorem may be given without recourse to Nevanlinna's formulas if we are content to establish the continuous variation of $F(z)$ merely for $|z| \leqq \rho<1$. This new proof depends on the continuity of $M$ considered as a function of the initial conditions and on the uniform continuity for $|z| \leqq \rho<1$ of functions analytic and of modulus not greater than $M+\epsilon$ for $|z| \leqq 1$. The proof is in spirit closely related to the proof of Theorem 2 below and can be easily supplied by the reader.

4. Approximation on a circle. In the notation of Theorem 1, we can prove at once another part of Theorem B:

THEOREM 2. Let the functions $F_{n}(z)$ analytic for $|z|<1$ take on, at the $k$ given points $P$ interior to $C:|z|=1$, the prescribed values for the functions and for their first $k_{P}$ derivatives. Then

$$
\lim _{n \rightarrow \infty}\left[\overline{\text { bound }} F_{n}(z),|z|<1\right]=M
$$

implies

$$
\lim _{n \rightarrow \infty} F_{n}(z)=F(z)
$$

for $|z|<1$, uniformly for $|z| \leqq \rho$, where $\rho$ is an arbitrary number less than unity. Here the function $F(z)$ is the function of Theorem 1 corresponding to the prescribed auxiliary conditions.

Interior to $C$ the functions $F_{n}(z)$ are uniformly bounded and hence form a normal family there. Then from any infinite set of the $F_{n}(z)$ can be extracted a subsequence which converges for $|z|<1$, uniformly for $|z| \leqq \rho<1$. The limit of such a subsequence is, by (4.1), a function whose modulus in $C$ is not greater than $M$. This limit function is analytic for $|z|<1$ and by the uniformity of the convergence takes on at the $k$ given points $P$ the prescribed values for itself and for its first $k_{P}$ derivatives, and hence by Theorem 1 coincides with $F(z)$. That is to say, from every infinite set of the $F_{n}(z)$ can be extracted a subsequence whose limit is $F(z)$, so the original sequence $F_{n}(z)$ has the limit $F(z)$ for $|z|<1$, and being uniformly bounded converges to the limit uniformly for $|z| \leqq \rho<1$.

The simplest cases of Theorem 2 are the cases where the prescribed auxiliary conditions are of the form

$$
F_{n}(0)=0, \quad F_{n}^{\prime}(0)=0, \cdots, F_{n}^{(k)}(0)=1 ;
$$

here Theorems 1 and 2 can be readily proved by elementary means, by studying $k ! F_{n}(z) / z^{k}$, and of course it turns out that the corresponding function $F(z)$ of Theorem 1 is $z^{k} / k$ !. 
A detailed study of the behavior of the functions $F_{n}(z)$ of Theorem 2 on the circumference $C$ is beyond the scope of the present paper, ${ }^{*}$ but it is perhaps of interest to give an elementary example indicating that the convergence in (4.2) need not be uniform for $|z|<1$. Our example shows in fact that $\lim _{n \rightarrow \infty} F_{n}(z)$ need not be continuous for $|z| \leqq 1$ even when the $F_{n}(z)$ are analytic for $|z| \leqq 1$. We set

$$
F_{n}(z)=e^{1 /[n(i-1)-1]+1 /(n+1)},
$$

which is analytic for $|z| \leqq 1$ and has the value unity at the origin. This prescription $\left(F_{n}(0)=1\right)$ is taken as the sole auxiliary condition on the functions $F_{n}(z)$, and the corresponding value of $M$ is unity. It is, moreover, clear that we have

$$
\lim _{n \rightarrow \infty} F_{n}(z)=\left\{\begin{array}{l}
1, z \neq 1, \\
\frac{1}{e}, z=1,
\end{array}\right.
$$

so that the limit function is discontinuous $\dagger$ not merely for $|z| \leqq 1$ but also for $|z|=1$. The absolute value of $F_{n}(z)$ is the exponential function of the real part of

$$
\frac{1}{n(z-1)-1}+\frac{1}{n+1}
$$

that is, the exponential function of

$$
\theta(x, y)=\frac{n(x-1)-1}{(n x-n-1)^{2}+n^{2} y^{2}}+\frac{1}{n+1} .
$$

On the circle $|z|=1$ we have

$$
\theta(x, y)=\frac{n(x-1)-1}{1-2\left(n^{2}+n\right)(x-1)}+\frac{1}{n+1},
$$

whose maximum value occurs for $x=-1$ :

$$
\theta(-1,0)=\frac{1}{n+1}-\frac{1}{2 n+1} .
$$

This expression approaches zero with $1 / n$, so (4.1) is indeed satisfied.

* See also $\$ 11$.

$\dagger$ It is not difficult to show that in Theorem 2

$$
\lim _{n \rightarrow \infty}\left|F_{n}(z)\right|<M
$$

is impossible on a point set $C$ of Lebesgue measure greater than zero. 
The particular sequence $F_{n}(z)$ may be easily replaced by a sequence of polynomials in $z$ which has the same convergence properties for $|z| \leqq 1$. In fact, we can prove that if $\left\{F_{n}(z)\right\}$ is an arbitrary sequence of functions analytic interior to a Jordan curve $C$ and continuous in the corresponding closed region $\bar{C}$, then there exists a sequence $\left\{P_{n}(z)\right\}$ of polynomials in z converging in $\bar{C}$ whenever the given sequence converges and to the same sum, diverging in $\bar{C}$ whenever the given sequence diverges, converging uniformly on any point sets belonging to $\vec{C}$ on which the given sequence converges uniformly, and such that we have $P_{n}(z)=F_{n}(z)$ in an arbitrary finite number of preassigned points $\alpha_{i}$ of $\bar{C}$. If the latter points are interior to $C$, we may require equality also of an arbitrary finite number of derivatives

$$
P_{n}^{(k)}\left(\alpha_{i}\right)=F_{n}{ }^{(k)}\left(\alpha_{i}\right) \quad\left(k=0,1, \cdots, k_{i}\right)
$$

at the preassigned points of $\bar{C}$.

There exist polynomials $P_{n}(z)$ such that we have

$$
\left|P_{n}(z)-F_{n}(z)\right|<\frac{1}{n},
$$

for $z$ in $\bar{C}$, and where we may choose $P_{n}^{(k)}\left(\alpha_{i}\right)=F_{n}^{(k)}\left(\alpha_{i}\right)$ as described.* The sequence $\left\{P_{n}(z)\right\}$ has all the properties required.

A simple transformation of the results considered in Theorem 2 will yield new results that we desire, parts of Theorem A.

THEOREM 3. Let $r(z)$ be a rational function of $z$ with all of its singularities interior to $C:|z|=1$. Of all functions $f(z)$ analytic for $|z|<1$, there exists $a$ unique function such that

$$
\overline{\substack{|z| \rightarrow 1 \\|z|<1}}_{\operatorname{bound}}|r(z)-f(z)|
$$

is least; this function $f(z)$ is rational, and $r(z)-f(z)$ is of constant modulus $M$ on $C$.

If the functions $f_{n}(z)$ are analytic for $|z|<1$, then

$$
\lim _{n \rightarrow \infty}\left[\overline{\substack{|z o u n d\\| z \mid<1}} \bar{d}\left|r(z)-f_{n}(z)\right|\right]=M
$$

implies

$$
\lim _{n \rightarrow \infty} f_{n}(z)=f(z)
$$

for $|z|<1$, uniformly for $|z|<\rho$, provided merely $\rho<1$.

* Compare the proof of Theorem 7 below. 
Let the given function be

$$
\begin{aligned}
r(z) & =\frac{a_{0} z^{\nu}+a_{1} z^{\nu-1}+\cdots+a_{\nu}}{\left(z-\alpha_{1}\right)\left(z-\alpha_{2}\right) \cdots\left(z-\alpha_{\mu}\right)} \\
& =A_{0}+\frac{A_{1}}{z-\alpha_{1}}+\frac{A_{2}}{z-\alpha_{2}}+\cdots+\frac{A_{\mu}}{z-\alpha_{\mu}},
\end{aligned}
$$

where all the $\alpha_{i}$ lie interior to the circle $C$. If the function $f(z)$ is analytic within $C$, continuous on and within $C$, then the function*

$$
F(z) \equiv[r(z)-f(z)] \frac{\left(z-\alpha_{1}\right)\left(z-\alpha_{2}\right) \cdots\left(z-\alpha_{\mu}\right)}{\left(1-\bar{\alpha}_{1} z\right)\left(1-\bar{\alpha}_{2} z\right) \cdots\left(1-\bar{\alpha}_{\mu} z\right)}
$$

is likewise analytic within $C$, continuous on and within $C$, and has on $C$ the same maximum absolute value $\dagger$ as $r(z)-f(z)$. In any case if $f(z)$ is analytic within $C$ (for the present $f(z)$ is subject to no other restriction), so also is $F(z)$, and it is true that

$$
\overline{\underset{|z|<1}{\operatorname{bound}}} F(z)=\overline{\substack{|z| \rightarrow 1 \\|z|<1}}[r(z)-f(z)]
$$

In the point $\alpha_{i}$ the function $F(z)$ has the value

$$
\begin{gathered}
\frac{a_{0} \alpha_{i}{ }^{\nu}+a_{1} \alpha_{i}{ }^{\nu-1}+\cdots+a_{\nu}}{\left(1-\bar{\alpha}_{1} \alpha_{i}\right)\left(1-\bar{\alpha}_{2} \alpha_{i}\right) \cdots\left(1-\bar{\alpha}_{\mu} \alpha_{i}\right)} \\
=A_{i} \frac{\left(\alpha_{i}-\alpha_{1}\right)\left(\alpha_{i}-\alpha_{2}\right) \cdots\left(\alpha_{i}-\alpha_{i-1}\right)\left(\alpha_{i}-\alpha_{i+1}\right) \cdots\left(\alpha_{i}-\alpha_{\mu}\right)}{\left(1-\bar{\alpha}_{1} \alpha_{i}\right)\left(1-\bar{\alpha}_{2} \alpha_{i}\right) \cdots\left(1-\bar{\alpha}_{\mu} \alpha_{i}\right)},
\end{gathered}
$$

which depends only on $r(z)$ and not in any way on $f(z)$. If two or more of the $\alpha_{i}$ in $r(z)$ are equal, that is to say, if $r(z)$ has poles of order higher than the first, then formula (4.5) requires some revision, and contains denominators of degree higher than the first. In this case not merely is the value of $F\left(\alpha_{i}\right)$ determined by (4.6) and independent of $f(z)$, but also the value of the first and possibly higher derivatives of $F(z)$ at $z=\alpha_{i}$, depending on the number of the $\alpha_{i}$ which coalesce. The reader can easily make the necessary modifications in the formulas given, to conform to this new situation. In every case the auxiliary conditions (4.8) are of precisely the kind we have been studying in Theorems 1 and 2.

* The case that $r(z)$ is a constant is trivial and henceforth excluded.

† For $z$ on $C$ we have $z \bar{z}=1$, so the expression

$$
\frac{z-\alpha_{1}}{1-\bar{\alpha}_{1} z}=\frac{z \bar{z}-\alpha_{1} \bar{z}}{\bar{z}-\bar{\alpha}_{1} z \bar{z}}=\frac{1-\alpha_{1} \bar{z}}{\bar{z}-\bar{\alpha}_{1}}
$$

equals the reciprocal of its conjugate and hence has the absolute value unity. 
Reciprocally, let the function $F(z)$ analytic interior to $C$ be given, which in the points $\alpha_{i}$ takes on the values (4.8). Let us write $F(z)$ in the form indicated by (4.6), where $r(z)$ is given by (4.5) as before. Then the function $f(z)$ defined by (4.6) is analytic interior to $C$ and is continuous on and within $C$ if $F(z)$ has that property; the maximum absolute value of $F(z)$ on $C$ is the same as that of $r(z)-f(z)$. In every case (4.7) is true. Here the formulas (4.5), (4.6), (4.8) are of course to be somewhat modified if $r(z)$ has multiple poles, but the modifications present no difficulty and are left to the reader.

The first part of Theorem 3 now follows immediately from Theorem 1, for we have transformed the situation of Theorem 3 into an equivalent situation in Theorem 1. The second part of Theorem 3 follows without difficulty from Theorem 2. In fact let us replace $f(z)$ in (4.6) by $f_{n}(z)$ and $F(z)$ by $F_{n}(z)$; this new equation

$$
F_{n}(z) \equiv\left[r(z)-f_{n}(z)\right] \frac{\left(z-\alpha_{1}\right)\left(z-\alpha_{2}\right) \cdots\left(z-\alpha_{\mu}\right)}{\left(1-\bar{\alpha}_{1} z\right)\left(1-\bar{\alpha}_{2} z\right) \cdots\left(1-\bar{\alpha}_{\mu} z\right)}
$$

is the definition of $F_{n}(z)$, whereas equation (4.6) is now considered to define the function $f(z)$ of Theorem 3 in terms of the function $F(z)$ of Theorem 1 for the auxiliary conditions (4.8). Then (4.4) implies

$$
\lim _{n \rightarrow \infty}\left[\overline{\text { bound }} F_{n \mid<1}(z)\right]=M \text {, }
$$

and hence by Theorem 2 implies

$$
\lim _{n \rightarrow \infty} F_{n}(z)=F(z)
$$

for $|z|<1$, uniformly for $|z| \leqq \rho$, where $F(z)$ is the function of Theorem 1 . This equation implies in turn

$$
\lim _{n \rightarrow \infty} f_{n}(z)=f(z),
$$

where $f(z)$ is defined by (4.6) in terms of the function $F(z)$ of Theorem 1 , and (4.9) holds for $|z|<1$, uniformly for $|z| \leqq \rho<1$, except in the neighborhoods of the points $\alpha_{i}$. But the functions $f_{n}(z), f(z)$ are analytic in the neighborhoods of those points as well as at the points $\alpha_{i}$, and equation (4.9), holding uniformly on small circles interior to $C$ surrounding the respective points $\alpha_{i}$, implies that (4.9) holds also interior to those small circles, so Theorem 3 is completely established.

It is not hard to obtain some information about the degree of the function $f(z)$ of Theorem 3. We find from (4.6),

$$
f(z) \equiv r(z)-\frac{\left(1-\bar{\alpha}_{1} z\right)\left(1-\bar{\alpha}_{2} z\right) \cdots\left(1-\bar{\alpha}_{\mu} z\right)}{\left(z-\alpha_{1}\right)\left(z-\alpha_{2}\right) \cdots\left(z-\alpha_{\mu}\right)} F(z) .
$$


The degree of $F(z)$ is less than $\mu$, and the degree of $r(z)$ is precisely $\mu$. When the two fractions in the right-hand member are reduced to a common denominator, the new fraction is of degree less than $2 \mu$. But the function $f(z)$ has no singularities interior to $C$, so the factors $z-\alpha_{i}$ are common to numerator and denominator, and the actual degree of $f(z)$ is less than $\mu$.

The simplest case of Theorem 3 is for approximation to the function

$$
r(z)=\frac{1}{z^{k}}, k>0 .
$$

Here the proof of the theorem can be given by elementary methods, and of course the function $f(z)$ is identically zero.

5. Some inequalities. It is reasonable to suppose that there exist inequalities, more explicit than Theorem 2, which give an upper limit for $\left|F_{n}(z)-F(z)\right|$ in terms of $\left\{\left[\overline{\text { bound }} F_{n}(z),|z|<1\right]-M\right\}$, indicating the fact that approach to zero of the latter quantity implies approach to zero of the former, if $z$ is properly restricted. Theorem 2 itself can be used to establish the existence of such inequalities, but we use the direct method based on the formulas of Nevanlinna. We shall treat in full detail only a special case, but shall indicate later the modifications necessary to treat the general case.

We state for reference what is essentially a particular case of the inequalities we propose to establish:

CaRathéodory's Lemma.* If the function $F(z)$ is analytic and in absolute value not greater than $N$ for $|z|<1$, then we have

$$
|F(z)-F(0)| \leqq|z| \frac{N^{2}-|F(0)|^{2}}{N-|F(0)| \cdot|z|} .
$$

If we set $N=|F(0)|+\nu$, this inequality can be written

$$
|F(z)-F(0)| \leqq|z| \frac{\nu[\nu+2|F(0)|]}{\nu+|F(0)|(1-|z|)} .
$$

It follows from (5.2) that if we have a sequence of functions $F_{n}(z)$ having the common value $F(0)$ for $z=0$, and if we have for $|z|<1$

$$
\left|F_{n}(z)\right| \leqq|F(0)|+\nu_{n}, \quad \lim _{n \rightarrow \infty} \nu_{n}=0,
$$

then we have $\lim _{n \rightarrow \infty} F_{n}(z)=F(0)$ uniformly for $|z| \leqq \rho<1$. This is a special case of Theorem 2, which together with the corresponding special case of

* Mathematische Annalen, vol. 72 (1912), p. 107. Pólya und Szegö, Aufgaben und Lehrsätze aus der Analysis, vol. I, pp. 139, 323. The Lemma there has the hypothesis $|F(z)|<N$, instead of $|F(z)| \leqq N$, but since it holds in the one case it holds also in the other. 
Theorem 1 can easily be proved without the aid of Carathéodory's Lemma. Nevertheless (5.2) is more explicit and for some purposes more useful, as we shall see, than Theorem 2 .

We treat now the special situation of Theorem 2 where $k=1$, and $P$ is the origin. The function $F(z)$ of Theorem 2 may be defined by the recurrent process of setting (Nevanlinna, loc. cit. p. 37; the formulas for this special case are those of Gronwall and Schur)

$$
\phi_{l}(z)=\frac{M^{2}}{z} \frac{\phi_{l-1}(z)-\phi_{l-1}(0)}{M^{2}-\bar{\phi}_{l-1}(0) \phi_{l-1}(z)}, \quad l=1,2, \cdots, p, \quad \phi_{0}(z) \equiv F(z)
$$

where $\phi_{p}(z)$ is a constant of modulus $M$ and where $p$ is the $k_{0}$ of Theorem $1 . *$ In exceptional cases it may occur that $\phi_{q}(z)$ is a constant (necessarily of modulus $M$ ) for some $q<p$. This involves a change in the notation but no essential change in the reasoning; the corresponding modifications here and below are left to the reader.

If we have now the functions $F_{n}(z)$ of Theorem 2 with the present auxiliary conditions, we set likewise

$$
\phi_{n l}(z)=\frac{M^{2}}{z} \frac{\phi_{n, l-1}(z)-\phi_{n, l-1}(0)}{M^{2}-\bar{\phi}_{n, l-1}(0) \phi_{n, l-1}(z)}, \quad \phi_{n 0}(z)=F_{n}(z) .
$$

It follows that we have

$$
\begin{gathered}
\phi_{l}(0)=\phi_{n l}(0), \quad l=1,2, \cdots, p ; n=1,2, \cdots ; \\
\left|\phi_{l}(z)\right| \leqq M \text { for }|z|<1, \quad l=0,1, \cdots, p .
\end{gathered}
$$

Direct computation yields

$$
\phi_{n l}(z)-\phi_{l}(z)=\frac{M^{2}}{z} \frac{\left[\phi_{l, n-1}(z)-\phi_{l-1}(z)\right]\left[M^{2}-\phi_{l-1}(0) \bar{\phi}_{l-1}(0)\right]}{\left[M^{2}-\bar{\phi}_{l-1}(0) \phi_{n, l-1}(z)\right]\left[M^{2}-\bar{\phi}_{l-1}(0) \phi_{l-1}(z)\right]} .
$$

Under our present hypothesis we have $\left|\phi_{l}(0)\right|<M$, for $l=0,1, \cdots, p-1$, so all of the factors $M^{2}-\phi_{l-1}(0) \bar{\phi}_{l-1}(0), M^{2}-\bar{\phi}_{l-1}(0) \phi_{l-1}(z), M^{2}-\bar{\phi}_{l-1}(0)$ $\cdot \phi_{n, l-1}(z)$ are bounded from zero, except possibly the last, and this is bounded from zero if $n$ is sufficiently great. We remark too that by virtue of the equation $\phi_{n, l-1}(0)=\phi_{l-1}(0)$, the function

$$
\frac{\phi_{n, l-1}(z)-\phi_{l-1}(z)}{z}
$$

is analytic for $|z|<1$, and hence has the upper limit of its modulus in $|z|<1$ not for $z=0$ but for $|z| \rightarrow 1$.

\footnotetext{
* We write, here and below, $\bar{\phi}_{k}(z)$ to denote the conjugate of $\phi_{k}(z)$.
} 
Equations (5.5) for $l=1,2, \cdots, p$ are to be used twice in deriving the inequalities we desire. Moreover it is the nature of the inequalities rather than their precise form that interests us, although the precise form can be readily obtained by the reader. We introduce the notation, under the hypothesis of Theorem 2 ,

$$
M+\epsilon_{n}=\overline{\text { bound }}\left|F_{n}(z)\right| \text { for }|z|<1,
$$

from which it follows that $\lim _{n \rightarrow \infty} \epsilon_{n}=0$.

By studying the transformation involved in (5.4), it is easily shown that the inequality $\left|\phi_{n, l-1}(z)\right| \leqq m_{n, l-1}$ implies

$$
\left|\phi_{n l}(z)\right| \leqq M^{2} \frac{m_{n, l-1}-\left|\phi_{l-1}(0)\right|}{M^{2}-m_{n, l-1}\left|\phi_{l-1}(0)\right|}, \text { for }|z|<1,
$$

if $m_{n, l-1}$ is sufficiently near to $M$, and we apply this formula to each of the functions $\phi_{n 1}(z), \phi_{n 2}(z), \cdots, \phi_{n p}(z)$ in turn. It is obvious that the right-hand member of (5.7) approaches $M$, as $m_{n, l-1}$ approaches $M$, and also that the derivative of the right-hand member with respect to $m_{n, l-1}$ for the value $m_{n, l-1}=M$ is finite, from which it follows that we have

$$
\left|\phi_{n l}(z)\right| \leqq M+M_{l}\left(m_{n, l-1}-M\right), \text { for }|z|<1,
$$

where $M_{l}$ is a suitably chosen number independent of $n$.

We use this inequality for $l=1,2, \cdots, p$ in succession, setting $m_{n l}=$ $M+M_{l}\left(m_{n, l-1}-M\right), m_{n 0}=M+\epsilon_{n}$, and derive

$$
\left|\phi_{n p}(z)\right| \leqq M+M^{\prime} \epsilon_{n}
$$

where $M^{\prime}$ is a suitably chosen number independent of $n$. Finally Carathéodory's Lemma informs us that we have

$$
\left|\phi_{n p}(z)-\phi_{p}(z)\right| \leqq M^{\prime \prime} \epsilon_{n} \text { for }|z| \leqq \rho<1,
$$

where $M^{\prime \prime}$ depends on $\rho$ but not on $n$; for it is true that $\phi_{p}(z) \equiv \phi_{p}(0)$. Immediate use of equation (5.5) for $l=p, p-1, \cdots, 1$ then gives inequalities similar to (5.8) where $p$ is replaced successively by $p-1, p-2, \cdots, 0$, and finally we have

$$
\left|F_{n}(z)-F(z)\right| \leqq M_{1}^{\prime} \epsilon_{n}, \text { for }|z| \leqq \rho<1,
$$

where $M_{1}^{\prime}$ depends on $\rho$ but not on $\epsilon_{n}$. All of these inequalities have been established only if $\epsilon_{n}$ is sufficiently small, but do hold under such restrictions, provided merely that $\epsilon_{n}$ is defined by (5.6).

We have given the derivation of (5.9) not for the most general situation of Theorem 2, but under the assumption that only the values of $F_{n}(z)$ and 
of its first $p$ derivatives are assigned at the origin. In the general case, the formulas given are to be used, and followed by others of the form

$$
\begin{aligned}
& \psi_{1}(z)=M^{2} \frac{\psi_{0}(z)-\psi_{0}\left(z_{0}\right)}{M^{2}-\bar{\psi}_{0}\left(z_{0}\right) \psi_{0}(z)}: \frac{z-z_{0}}{1-\bar{z}_{0} z}, \\
& \psi_{n 1}(z)=M^{2} \frac{\psi_{n 0}(z)-\psi_{n 0}\left(z_{0}\right)}{M^{2}-\bar{\psi}_{n 0}\left(z_{0}\right) \psi_{n 0}(z)}: \frac{z-z_{0}}{1-\bar{z}_{0} z},
\end{aligned}
$$

which correspond to (5.3) and (5.4) respectively. We have

$$
\psi_{0}(z)=F(z) \text { or } \phi_{p}(0), \quad \psi_{n 0}(z)=F_{n}(z) \text { or } \phi_{n p}(z),
$$

and of course we have $\psi_{0}\left(z_{0}\right)=\psi_{n 0}\left(z_{0}\right)$. We compute now the analogue of (5.5):

$$
\psi_{n 1}(z)-\psi_{1}(z)=\frac{M^{2}}{\frac{z-z_{0}}{1-\bar{z}_{0} z}} \frac{\left[\psi_{n 0}(z)-\psi_{0}(z)\right]\left[M^{2}-\psi_{0}\left(z_{0}\right) \bar{\psi}_{0}\left(z_{0}\right)\right]}{\left[M^{2}-\bar{\psi}_{0}\left(z_{0}\right) \psi_{0}(z)\right]\left[M^{2}-\bar{\psi}_{n 0}\left(z_{0}\right) \psi_{n 0}(z)\right]} .
$$

By reasoning similar to that already given we now obtain (5.9) as before, provided that $\epsilon_{n}$ is sufficiently small. This holds whether we have a single application of formulas (5.10) and (5.11), or repeated application, and also with or without further repeated application of formulas of the type (5.3) and (5.4).

This gives us indeed the most general situation under Theorem 2 , for we can first transform an arbitrary point $P$ to the origin, and use (5.3) and (5.4) to account for prescribed values of function and derivatives at $P$; then we can transform a second point $P$ to the origin and account for the prescribed values at this new point by means of (5.10) and (5.11) followed if necessary by (5.3) and (5.4), and so on. The inequalities we are using and deriving are in character unaltered by transformations of the types used.

Inequality (5.9) contains the essence of Theorem 2.

Julia has recently used* a special case of (5.9) in a study to which more detailed reference will be made later.

The discussion just given can be applied with ease by the methods of $\S 4$ to the situation of Theorem 3. We introduce the notation

$$
M+\epsilon_{n}=\overline{\text { bound }}\left|r(z)-f_{n}(z)\right| \text { for }|z| \rightarrow 1,|z|<1 \text {. }
$$

The functions $F_{n}(z)$ are defined as in (4.6):

$$
F_{n}(z) \equiv\left[r(z)-f_{n}(z)\right] \frac{\left(z-\alpha_{1}\right)\left(z-\alpha_{2}\right) \cdots\left(z-\alpha_{\mu}\right)}{\left(1-\bar{\alpha}_{1} z\right)\left(1-\bar{\alpha}_{2} z\right) \cdots\left(1-\bar{\alpha}_{\mu} z\right)}
$$

\footnotetext{
* Annales de l'École Normale Supérieure, (3), vol. 44 (1927), pp. 289-316.
} 
thus (5.6) may equally well be used as a definition of $\epsilon_{n}$. For $\epsilon_{n}$ sufficiently small, we have shown (5.9) to be valid. Direct computation by means of (4.6) and (5.12) then implies

$$
\left|f_{n}(z)-f(z)\right| \leqq M_{2}^{\prime} \epsilon_{n} \text { for }|z| \leqq \rho<1 .
$$

Inequality (5.13) follows from (4.6) and (5.12) first for $|z|=\rho$, where $\left|\alpha_{i}\right|<\rho$, but holding on the circle $|z|=\rho$ holds also within this circle as well.

6. Tchebycheff polynomials. A special sequence of approximating functions $\left\{f_{n}(z)\right\}$ or $\left\{F_{n}(z)\right\}$ which has interesting properties will now be studied.

If $\phi(z)$ is a continuous function defined on the closed limited point set $C$ of the $z$-plane, then there exists* a unique polynomial $\pi_{n}(z)$ of degree $\dagger n$ such that

$$
\max \left|\phi(z)-\pi_{n}(z)\right|, z \text { on } C,
$$

is less than the corresponding expression

$$
\max \left|\phi(z)-p_{n}(z)\right|, z \text { on } C,
$$

for any other polynomial $p_{n}(z)$ of degree $n$. The polynomial $\pi_{n}(z)$ is called the Tchebycheff polynomial of degree $n$ for approximation to $\phi(z)$ on $C$, and has many important and well known properties. The following theorem, whose various parts are due to various writers, $\ddagger$ will yield us the results on Tchebycheff polynomials contained in Theorems A and B.

Theorem 4. Let $C$ be a closed limited point set (not a single point) of the $z$-plane whose complementary set with respect to the entire plane is simply connected. Denote by $C_{R}$ the image in the z-plane of the circle $|w|=R>1$ when the set complementary to $C$ is mapped onto the exterior of the unit circle in the $w$-plane so that the points at infinity correspond to each other. Then if $f(z)$ is analytic in the closed interior of $C_{R}$, there exist polynomials $p_{n}(z)$ of respective degrees $n=0,1,2, \cdots$, such that we have

$$
\left|f(z)-p_{n}(z)\right| \leqq \frac{K}{R^{n}}, z \text { on } C, n=0,1,2, \cdots,
$$

where $K$ is independent of $n$ and $z$.

\footnotetext{
* See $\$ 10$ below. The theorem (due to Tonelli) assumes that $C$ contains at least $n+1$ distinct points.

$\dagger$ That is, $\pi_{n}(z)$ can be written in the form $a_{0} z^{n}+a_{1} z^{n-1}+\cdots+a_{n}$.

$\ddagger$ References are given by Walsh, Münchner Berichte, 1926, pp. 223-229. In this theorem and below we tacitly assume that if the function $f(z)$ is not defined on the entire point set considered, then the function is to be defined on the new points by analytic extension, or (what amounts to the same thing) by the convergent sequence of polynomials.
} 
If the polynomials $p_{n}(z)$ are given so that (6.3) is valid for $z$ on $C$, where $f(z)$ is defined merely on $C$, then the sequence $\left\{p_{n}(z)\right\}$ converges interior to $C_{R}$, $R>1$, uniformly on any closed point set interior to $C_{R}$, and $f(z)$ is analytic interior to $C_{R}$. Moreover, an inequality of the form

$$
\left|f(z)-p_{n}(z)\right| \leqq K_{1}\left(\frac{R_{1}}{R}\right)^{n} \quad(n=0,1,2, \cdots)
$$

is valid for $z$ on and within an arbitrary $C_{R_{1}}, R_{1}<R$.

In particular, if $f(z)$ is an entire function, the polynomials $p_{n}(z)$ exist so that for an arbitrary $R>1$ and for suitable $K$ (depending on $R$ ), the inequality (6.3) holds, and the sequence $\left\{p_{n}(z)\right\}$ converges over the entire plane, uniformly on any closed point set.

If in Theorem 4 the polynomials $p_{n}(z)$ are given so that (6.3) holds merely for $n$ sufficiently large, say $n \geqq N$, then arbitrary polynomials can be chosen as the $p_{n}(z), n<N$, if those polynomials are not already defined, and for a suitably modified $K$ the inequality (6.3) will hold for all values of $n$. That is, it is still true that the sequence $\left\{p_{n}(z)\right\}$ converges interior to $C_{R}$, uniformly on an arbitrary point set interior to $C_{R}$.

With the same notation as in Theorem 4, we can now prove* easily

TheOREM 5. If $f(z)$ is analytic interior to $C_{R}$ and if $\pi_{n}(z)$ is the Tchebycheff polynomial for approximation to $f(z)$ on $C$, then we have

$$
\left|f(z)-\pi_{n}(z)\right| \leqq \frac{K_{1}}{R_{1}^{n}}, \text { z on } C, R_{1}<R,
$$

and the sequence $\pi_{n}(z)$ converges throughout the interior of $C_{R}$, uniformly on any closed point set interior to $C_{R}$.

Theorem 5 is a direct consequence of Theorem 4 . For if an arbitrary $R_{1}<R$ is given, there exist polynomials $p_{n}(z)$ of respective degrees $n=0,1,2$, $\cdots$, such that we have

$$
\left|f(z)-p_{n}(z)\right| \leqq \frac{K_{1}}{R_{1}{ }^{n}}, z \text { on } C .
$$

Since this inequality is valid for the polynomials $p_{n}(z)$ it is likewise valid for the Tchebycheff polynomials $\pi_{n}(z)$, so that (6.4) holds. By virtue of Theorem 4 , Theorem 5 now follows as stated. We note that if $f(z)$ has a singularity

* Theorem 5 was proved by Faber in the special case that $C$ is a region bounded by an analytic Jordan curve, by a method quite different from the present one, Crelle's Journal, vol. 150 (1920), pp. 79-106; p. 105. The present method applies also in the case of harmonic functions and harmonic polynomials; see Walsh, Bulletin of the American Mathematical Society, vol. 35 (1929), pp. 499-544; p. 513 . 
on $C_{R}$, then (6.4) can hold for $z$ on $C$ and with $R_{1}>R$ for no sequence of polynomials $\left\{\pi_{n}(z)\right\}$.

When the function $f(z)$ is analytic on $C$, it is immaterial whether we consider the Tchebycheff polynomial for approximation to $f(z)$ on $C$ or merely on the boundary of $C$. For the functions whose absolute values appear in (6.1) and (6.2) take on their greatest moduli on the boundary. It is natural, then, to restrict ourselves to point sets $C$ which are boundaries of regions, and this is forced upon us if we consider, as we shall now do, functions with singularities interior to those regions.

THEOREM 6. Let $r(z)$ be a rational function of $z$ with all of its singularities interior to $C:|z|=1$, and let $\pi_{n}(z)$ be the Tchebycheff polynomial of degree $n$ for approximation to $r(z)$ on $C$. Let the function $f(z)$ (of Theorem 3 ) be analytic for $|z|<R>1$ but have a pole on the circle $|z|=R$. Then the sequence $\left\{\pi_{n}(z)\right\}$ converges to the limit $f(z)$ for $|z|<R$, uniformly for $|z|<R_{1}<R$.

It is to be noted that $f(z)$ is analytic for $|z|=1$, having on that circle the modulus $M$, and hence is analytic for $|z|$ less than some $R$ which is greater than unity. The, Tchebycheff polynomials $\pi_{n}^{\prime}(z)$ for approximation to $f(z)$ on $C$ satisfy the inequality

by Theorem 5 . Thus we can write

$$
\left|f(z)-\pi_{n}^{\prime}(z)\right| \leqq \frac{K}{R_{1}^{n}}, z \text { on } C,
$$

$$
\left|r(z)-\pi_{n}^{\prime}(z)\right| \leqq|r(z)-f(z)|+\left|f(z)-\pi_{n}^{\prime}(z)\right| \leqq M+\frac{K}{R_{1}^{n}},
$$

for $z$ on $C$. This inequality, holding for the polynomial $\pi_{n}^{\prime}(z)$, yields us the inequality

$$
\left|r(z)-\pi_{n}(z)\right| \leqq M+\frac{K}{R_{1}{ }^{n}}, z \text { on } C,
$$

which by (5.13) for $\epsilon_{n}=K / R_{1}^{n}$ yields

$$
\left|f(z)-\pi_{n}(z)\right| \leqq \frac{K^{\prime}}{R_{1}^{n}}
$$

for $z$ on the circle $C^{\prime}:|z|=\rho$, where $\rho$ is less than unity but is otherwise arbitrary. We apply Theorem 4 to the sequence $\left\{\pi_{n}(z)\right\}$ and to this circle $C^{\prime}$, which yields the convergence of the sequence $\left\{\pi_{n}(z)\right\}$ for $|z|<\rho R_{1}$, uniformly for $|z|<\rho^{\prime}<\rho R_{1}$. The numbers $\rho$ and $R_{1}$ are arbitrary, subject merely to the restriction of being respectively less than 1 and $R$, so Theorem 6 follows in its entirety.

Theorem 6 is obviously related to Theorem 3, but gives a more specific result concerning a more specific sequence of functions. There is an analogous 
result related to Theorem 2, which involves the notion of Tchebycheff polynomials with auxiliary conditions.

We consider as before a function $\phi(z)$ continuous on a closed limited point set $C$. We consider all polynomials $p_{n}(z)$ of degree $n$ which take on prescribed values at $m$ points $P$ not belonging to $C$; such polynomials surely exist if $m$ is not greater than $n+1$. If the number of points of $C$ is sufficiently large (see $\$ 10)$ it can be shown that there exists a unique polynomial $\pi_{n}(z)$ belonging to this set of polynomials such that (6.1) is less than the expression (6.2) for any other polynomial $p_{n}(z)$ of the set. We shall call $\pi_{n}(z)$ the Tchebycheff polynomial for approximation to $\phi(z)$ on $C$ with the prescribed auxiliary conditions. It is permitted to consider the points $P$ not to be all distinct, that is, the auxiliary conditions may involve not merely the values of the polynomials $p_{n}(z)$ and $\pi_{n}(z)$ at the given points $P$, but also the values of the polynomials and their first $k_{P}$ derivatives at the points $P$.

The situation of Theorem 2 corresponds to approximation on the circle $C$ to the function $\phi(z) \equiv 0$, with the prescribed auxiliary conditions.

THEOREM 7. Let the function $F(z)$ of Theorem 1 be analytic for $|z|<R>1$, but have a pole for which $|z|=R$. Then the sequence of Tchebycheff polynomials $\pi_{n}(z)$ (for approximation on $C$ to the function zero with the prescribed auxiliary conditions) converges to the function $F(z)$ for $|z|<R$, uniformly for $|z|<R_{1}<R$.

There exist, by Theorem 4, polynomials $p_{n}(z)$ of respective degrees $n=0$, $1, \cdots$, such that we have

$$
\left|F(z)-p_{n}(z)\right| \leqq \frac{K}{R_{1}^{n}}, z \text { on } C,
$$

where $R_{1}<R$ is arbitrary. For $n$ sufficiently large there exist polynomials $q_{n}(z)$ of respective degrees $n$, equal in the prescribed points $P$ to the functions $F(z)-p_{n}(z)$, and such that*

$$
\left|q_{n}(z)\right| \leqq \frac{K^{\prime}}{R_{1}^{n}}, z \text { on } C .
$$

* See Walsh, these Transactions, vol. 30 (1928), pp. 307-332; Lemma, p. 319. The proof is there given only for the case $k_{P}=0$, but the general case can be treated in the same way. This same proof of (6.6) holds also for approximation to the real function $F(x)$ of the real variable $x$ on an interval $a \leqq x \leqq b$, and shows that the introduction of auxiliary conditions, requiring that the approximating polynomials $\pi_{n}(x)$ take on values of the function to be approximated at a finite number of preassigned points of the interval $(a, b)$, does not alter the degree of convergence of the sequence of Tchebycheff polynomials, if that is of the form corresponding to an inequality

$$
\left|F(x)-\pi_{n}(x)\right| \leqq M \cdot \epsilon(n),
$$

where $M$ is independent of $n$ and $\epsilon(n)$ approaches zero with $1 / n$. Compare Jackson, Bulletin of the American Mathematical Society, vol. 32 (1926), pp. 259-262, who proves the corresponding fact for approximation to continuous functions by trigonometric polynomials with auxiliary conditions. If it is a question merely of establishing (6.6) in the present case, the treatment given in $\$ 12.2$ of the present paper suffices. 
Hence we have for $n$ sufficiently large

$$
\left|F(z)-\left[p_{n}(z)+q_{n}(z)\right]\right| \leqq \frac{K+K^{\prime}}{R_{1}^{n}}, z \text { on } C ;
$$

at each point $P$ we have

$$
F(z)=p_{n}(z)+q_{n}(z),
$$

so the polynomial $p_{n}(z)+q_{n}(z)$, like the function $F(z)$, satisfies the prescribed auxiliary conditions. Thus we have for the Tchebycheff polynomials

$$
\left|F(z)-\pi_{n}(z)\right| \leqq \frac{K+K^{\prime}}{R_{1}{ }^{n}}, z \text { on } C,
$$

and Theorem 7 follows from Theorem 4.

The notion of Tchebycheff polynomial is of course far more general than we have considered here. Let $C$ be any point set, and $F(z)$ a function defined on $C$, to be approximated on $C$ by linear combinations of functions of the given set $\left\{\chi_{n}(z)\right\}$. The function of the form

$$
\Sigma_{n}(z)=a_{1} \chi_{1}(z)+\cdots+a_{n} \chi_{n}(z)
$$

such that

$$
\overline{\text { bound }}\left|F(z)-\Sigma_{n}(z)\right|, z \text { on } C \text {, }
$$

is least is called the Tchebycheff polynomial $\Sigma_{n}^{\prime}(z)$ of order $n$ for approximation to $F(z)$ on $C$. It is also possible not to admit all functions of form (6.7) into consideration, but to require for instance auxiliary conditions as in the present paragraph.

The Tchebycheff polynomial $\Sigma_{n}(z)$ may or may not exist, and if it exists may or may not be unique.* It is always true, however, that the sequence

$$
M_{n}=\overline{\text { bound }}\left|F(z)-\Sigma_{n}^{\prime}(z)\right|, z \text { on } C,
$$

decreases monotonically and hence approaches a limit, for the function $\Sigma_{n}^{\prime}(z)$ may also be considered a function $\Sigma_{n+1}(z)$. It is also true that if the lower limit of (6.8) for all admissible functions $\Sigma_{n}(z), n=1,2, \cdots$, is a number $M$, then we have

$$
\lim _{n \rightarrow \infty} M_{n}=M \text {. }
$$

For $\lim _{n \rightarrow \infty} M_{n}$ can be greater than no expression (6.8).

7. Regions more general than circles; approximation with auxiliary conditions. Theorems 1-3 hold with only obvious changes for simply connected regions $\dagger$ other than circles. The new theorems are proved from the old ones by means of conformal mapping of the new regions on the circle.

* Compare Haar, Mathematische Annalen, vol. 78 (1918), pp. 294-311.

$\dagger$ A region is an open connected point set. 
THEOREM 8. Let $B$ be an arbitrary simply connected region of the w-plane with the boundary $C$. Among all functions analytic interior to $B$ each of which at $k$ given points $P$ of $B$ takes on preassigned values for itself and its first $k_{P}$ derivatives, there exists a unique function $G(w)$ which has the smallest least upper bound for its modulus in $B$. The modulus of this function $G(w)$ approaches a constant value $M$ as w remaining in $B$ approaches $C$.

If the functions $G_{n}(w)$ analytic in $B$ satisfy the auxiliary conditions at the $k$ points $P$, then

$$
\lim _{n \rightarrow \infty}\left[\overline{\text { bound }} G_{n}(w), w \text { in } B\right]=M
$$

implies $\lim _{n \rightarrow \infty} G_{n}(w)=G(w)$ for $w$ in $B$, uniformly for $w$ on any closed point set interior to $B$.

Theorem 8 follows directly from Theorems 1 and 2, for under conformal mapping of $B$ onto the unit circle in the $z$-plane, a function which takes on prescribed values at definite points of $B$ corresponds to a function which takes on prescribed values at definite points in the interior of the unit circle, and conversely. Prescription of a certain number of derivatives at a point in the $w$-plane implies prescription of the same number of derivatives at the corresponding point of the $z$-plane, and conversely. Moreover, if $w$ in $B$ approaches $C$, then $z$, lying in the unit circle, approaches the circumference, and conversely.

A degenerate case occurs here if the region $B$ is the entire $w$-plane, or the entire plane except for a single point. In this case the conformal map is impossible. If there exists a constant satisfying the prescribed auxiliary conditions, Theorem 8 is valid as stated, $M$ being the absolute value of this constant. If no such constant exists, there is no function which satisfies the auxiliary conditions whose modulus in $B$ has a finite upper bound.

If the region $B$ is bounded by an analytic Jordan curve $C$, a more explicit result than Theorem 8 can be established for the Tchebycheff polynomials with the prescribed auxiliary conditions; this new result is a generalization of Theorem 7 .

THEOREM 9. If in Theorem 8 the region $B$ is the interior of an analytic Jordan curve $C$, then there exists some $C_{R}$ such that the function $G(w)$ is analytic for winterior to $C_{R}$ but has a singularity on $C_{R}$. Then the sequence of Tchebycheff polynomials $\left\{\tau_{n}(w)\right\}$ (for approximation on $C$ to the function zero with the prescribed auxiliary conditions) converges to the function $G(w)$ for w interior to $C_{R}$, uniformly for w on an arbitrary closed point set interior to $C_{R}$.

The proof here follows directly the proof of Theorem 7 and is omitted.

A less explicit result can be obtained for much more general regions. 
THEOREM 10. If in Theorem 8 the finite region $B$ is such that its boundary is also the boundary of an infinite region, then the sequence $\left\{\tau_{n}(w)\right\}$ of Tchebycheff polynomials for approximation to the function zero on $C$ with the given auxiliary conditions converges to the function $G(w)$ in $B$, uniformly on any closed point set interior to $B$.

Let $B_{\mu}$ be a sequence of closed regions bounded by analytic Jordan curves $C_{\mu}$ converging monotonically to $C$. That is, $B$ and $C_{\mu+1}$ are interior to $C_{\mu}$, and no point not belonging to the closed region $B$ lies interior to all the $C_{\mu}$ unless such a point cannot be joined to the point at infinity by a broken line which does not meet $C$. Let $\tau_{\mu n}(w)$ be the Tchebycheff polynomial of degree $n$ for the region $B_{\mu}$, that is, having the least maximum modulus in that closed region and satisfying the given auxiliary conditions; these auxiliary conditions are simply that the polynomial should take on certain values at the given points $P$ interior to $B$ and do not depend on $\mu$. Let $M_{\mu n}$ denote the maximum modulus of $\tau_{\mu n}(w)$ in $B_{\mu}$-that is, on $C_{\mu}$.

We have the relation

$$
M_{\mu+1, n} \leqq M_{\mu n},
$$

for $\tau_{\mu n}(w)$ is a polynomial of degree $n$ which satisfies the given auxiliary conditions, and its maximum modulus on $C_{\mu+1}$ is less than or equal to $M_{\mu n}$, its maximum modulus on $C_{\mu}$, since $C_{\mu+1}$ is interior to $C_{\mu}$; the equality can occur only if $\tau_{\mu n}(w)$ is a constant. Hence the maximum modulus of $\tau_{\mu+1, n}(w)$ on $C_{\mu+1}$ is less than $M_{\mu n}$, or in case $\tau_{\mu+1, n}(w)$ is the same as $\tau_{\mu n}(w)$ and a constant, is equal to $M_{\mu n}$. We have also the relation

$$
M_{\mu, n+1} \leqq M_{\mu n}
$$

for $\tau_{\mu n}(w)$ can be considered a polynomial of degree $n+1$, and satisfies the auxiliary conditions.

The region $B$ is the kernel of the regions $B_{\mu}$, and it follows* from the properties of the conformal map and from $\$ 3$ that as $\mu$ becomes infinite the functions $G_{\mu}(w)$ (of Theorem 8) corresponding to the regions $B_{\mu}$ approach the function $G(w)$ throughout $B$, uniformly on any closed point set interior to $B$. For map both $B$ and $B_{\mu}$ onto the unit circle in the $z$-plane so that a definite point and direction in the w-plane correspond to one and the same point and direction in the $z$-plane. The difference between the mapping functions is small, uniformly in an arbitrary closed region interior to $B$, and can be

* Carathéodory, Mathematische Annalen, vol. 72 (1912), pp. 107-144, chapter III. 
made as small as desired by choosing $\mu$ sufficiently large, ${ }^{*}$ and the same is true of the derivatives of the mapping functions. The auxiliary conditions for the Tchebycheff polynomials thus differ only slightly in the two cases, approximation in $B$ and in $B_{\mu}$, when we interpret the problem in the $z$-plane after mapping these respective regions on the unit circle. We now obtain the functions $G(w)$ and $G_{\mu}(w)$ by mapping the unit circle on $B$ and $B_{\mu}$ respectively, and these mapping functions differ but little from each other on an arbitrary closed point set interior to the unit circle; the difference can be made uniformly and arbitrarily small by choosing $\mu$ sufficiently large. Hence by $\S 3$ the difference between the functions $G(w)$ and $G_{\mu}(w)$ can be made as small as desired on an arbitrary closed point set interior to $B$, by choosing $\mu$ sufficiently large. It follows likewise from $\S 3$ that

$$
\lim _{\mu \rightarrow \infty} M_{\mu}=M,
$$

where $M$ and $M_{\mu}$ are respectively the maximum moduli of $G(w)$ and $G_{\mu}(w)$ in $B$ and in $B_{\mu}$.

By virtue of the two relations (7.1) and (7.2), it follows that $\lim _{\mu, n \rightarrow \infty} M_{\mu n}$ exists, and that this limit is equal to

$$
\lim _{\mu \rightarrow \infty}\left[\lim _{n \rightarrow \infty} M_{\mu n}\right] .
$$

By the method of proof of Theorem 9 we have $\lim _{n \rightarrow \infty} M_{\mu n}=M_{\mu}$, so by (7.3) we have (7.4) equal to $M$. Hence, by the monotonic character of the sequence $M_{\mu n}$, we have also

$$
\lim _{n \rightarrow \infty}\left[\lim _{\mu \rightarrow \infty} M_{\mu n}\right]=M .
$$

* The condition that the boundary of $B$ should also be the boundary of an infinite region is precisely the form of condition necessary for the application of Carathéodory's theorem, when we approximate $B$ externally, as here with the regions $B_{\mu}$. The region $B$ may still be fairly complicated and in particular its boundary may separate the plane into more than two regions. The region $B$ may be, for example, a strip which is closed at one end and approaches a circle by winding about it externally infinitely often. It is because the boundary of $B$ may separate the plane into more than two regions and because of the corresponding difficulties after conformal mapping that the function $f(z)$ of Theorem $A$ is so restricted as to have no singularities except in $B$ and in the infinite region of which $C$ is the boundary; compare Theorem 15 below.

Compare Julia, loc. cit., chapter III.

The Tchebycheff polynomial with auxiliary conditions for approximation on an arbitrary closed limited point set $C$, to a function $F(z)$ continuous on $C$ and analytic in the interior points of $C$, is precisely the same as the Tchebycheff polynomial with those auxiliary conditions for approximation on the boundary $C^{\prime}$ of the infinite region $B^{\prime}$ consisting of all points which can be joined with the point at infinity by broken lines not meeting $C$. For the maximum on $C$ of such a function as $\left|F(z)-\pi_{n}(z)\right|$, where $\pi_{n}(z)$ is the Tchebycheff polynomial, occurs on $C^{\prime}$. Special cases of this fact are indicated by Julia for his particular auxiliary conditions. 
The quantity $M_{n}^{\prime}$ here in square brackets is equal to the maximum modulus $M_{n}^{\prime \prime}$ on $C$ of $\tau_{n}(w)$. For if we had $M_{n}^{\prime \prime}<M_{n}^{\prime}$, we should be able to find some region $B_{\mu}$ for which the Tchebycheff polynomial $\tau_{\mu n}(w)=\tau_{n}(w)$ likewise had in $B_{\mu}$ a maximum modulus less than $M_{n}^{\prime}$, in contradiction with (7.1). The relation $M_{n}^{\prime}<M_{n}^{\prime \prime}$ is likewise impossible, for the maximum modulus of $\tau_{\mu n}(w)$ in $B$ is less than or equal to $M_{\mu n}$, for $B$ is interior to $C_{\mu}$, hence $M_{n}^{\prime \prime}$ is less than or equal to $M_{\mu n}$.

The relation (7.5) can be written

$$
\lim _{n \rightarrow \infty} M_{n}^{\prime \prime}=M,
$$

which by virtue of Theorem 8 gives now Theorem 10 . We might well state a more general result, for we have

$$
\lim _{\mu, n \rightarrow \infty} M_{\mu n}=M,
$$

so that we have proved the existence of the double limit

$$
\lim _{\mu, n \rightarrow \infty} \tau_{\mu n}(w)=G(w)
$$

throughout $B$, uniformly on any closed point set interior to $B$.

The most interesting auxiliary conditions in connection with Theorems $8-10$ are

$$
\tau_{n}(0)=0, \quad \tau_{n}^{\prime}(0)=1,
$$

where the origin $w=0$ is interior to $C$. Map the region $B$ onto the interior of the unit circle $|z| \leqq 1$ by means of the function $w=\phi(z), z=\psi(w)$, so that $w=0$ corresponds to $z=0$; the auxiliary conditions are equivalent to $\tau_{n}[\phi(0)]=0$, $\tau_{n}^{\prime}[\phi(0)]=1$. This second relation is, by the formula

equivalent to

$$
\frac{d \tau_{n}}{d w}=\frac{d \tau_{n}}{d z} \frac{d z}{d w}
$$

$$
\frac{d \tau_{n}}{d z}=a, \text { some constant not zero. }
$$

The problem of approximation in $B$ is equivalent to the problem of approximation in the unit circle of the $z$-plane by functions of the form $F_{n}(z)=a z+a_{2} z^{2}+a_{3} z^{3}+\cdots$, where $a$ is prescribed but the values $a_{i}$ are not prescribed; the modulus of $F_{n}(z)$ for $|z|<1$ is not greater than

$$
\overline{\text { bound }}|a|<1=a_{2} z+a_{3} z^{2}+\cdots \mid
$$

whose minimum value (compare Carathéodory's Lemma) is of course $|a|$. 
The corresponding function $F(z)$ is $a z$, and the function $G(w)$ is $a z=a \psi(w)$, which maps $B$ onto a circle in the w-plane.

Our general problem of Theorems 8-10 for the particular auxiliary conditions (7.6) has been studied in detail by Julia (loc. cit.), who has obtained for that case the principal results, with the exception of Theorem 9, that we have obtained in the more general case.

8. Regions more general than circles; approximation to a rational function. Just as we have extended Theorems 1 and 2 to the case of regions more general than circles, we shall now similarly extend Theorem 3:

THEOREM 11. Let $B$ be an arbitrary simply connected region of the w-plane with the boundary $C$, and let $s(w)$ be a rational function of $w$ with all of its singularities interior to $B$. Of all functions $g(w)$ analytic interior to $B$, there exists a unique function $g(w)$ such that

$$
\text { bound }|s(w)-g(w)| \text { for w in B approaching } C
$$

is least; the function $s(w)-g(w)$ is of constant modulus $M$ on $C$,- that is, the modulus of $s(w)-g(w)$ approaches a constant value as $w$ in $B$ approaches $C$.

If the functions $g_{n}(w)$ are analytic for $w$ in $B$, then

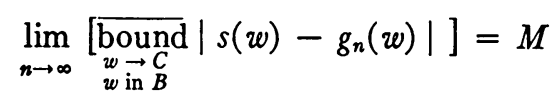

implies

$$
\lim _{n \rightarrow \infty} g_{n}(w)=g(w)
$$

for w in $B$, uniformly for w on any closed point set in $B$.

This theorem follows without difficulty from Theorem 3 by conformal mapping of the interior of $B$ onto the interior of the unit circle in the $z$-plane. Under the map the rational function $s(w)$ corresponds to a function of $z$ :

$$
s(w)=r(z)-s_{1}(z),
$$

where $r(z)$ is a rational function of $z$ and $s_{1}(z)$ is analytic for $|z|<1$. There exists a function $f(z)$ for which

$$
\varlimsup_{\substack{|z| \rightarrow|| z \mid<1}}|r(z)-f(z)|
$$

is least. Under the conformal map, a function analytic interior to $B$ corresponds to a function analytic for $|z|<1$ and conversely, and $|z| \rightarrow 1$ implies $w \rightarrow C$ and conversely, so this upper bound is the same as (8.1), if we set

$$
s_{1}(z)+g(w)=f(z) .
$$


A unique minimizing function $f(z)$ leads to a unique minimizing function $g(w)$ and conversely. We can similarly identify (4.4) and (8.2) by setting

$$
s_{1}(z)+g_{n}(w)=f_{n}(z)
$$

equation (8.2) implies $\lim _{n \rightarrow \infty} f_{n}(z)=f(z)$, which implies $\lim _{n \rightarrow \infty} g_{n}(w)=g(w)$, uniformly as stated, so Theorem 11 is completely established.

A degenerate case of Theorem 11 deserves special mention, when $B$ is the entire $w$-plane or the entire plane except for a single point $w=\alpha$. The first case has no meaning, for (8.1) of necessity refers to the boundary $C$ and here $C$ is non-existent. The second case is not difficult if we interpret (8.1) literally. All the functions $g(w)$ which concern us are constants, and the least (8.1) is given by setting $g(w) \equiv s(\alpha)$, so that (8.1) is zero. The second part of the theorem is also exact. In the future we shall not mention the possibility of the degeneracy of the region $B$; the reader can easily treat the new situation, for instance in Theorem 14.

In a special case we have a result more specific than Theorem 11:

THEOREM 12. If in Theorem 11 the region $B$ is the interior of an analytic Jordan curve $C$, the function $g(w)$ is analytic for $w$ interior to some $C_{R}$, but has a singularity on $C_{R}$. Then the sequence of Tchebycheff polynomials $\omega_{n}(w)$ for approximation on $C$ to the function $s(w)$ converges to the function $g(w)$ for $w$ interior to $C_{R}$, uniformly for $w$ on an arbitrary closed point set interior to $C_{R}$.

In the notation just used, we have by Theorem 4 the existence of a set of polynomials $\omega_{n}^{\prime}(w)$ of respective degrees $n$ such that

$$
\left|g(w)-\omega_{n}^{\prime}(w)\right| \leqq \frac{M^{\prime}}{R_{1}^{n}}, \text { for } w \text { on } B+C,
$$

where $R_{1}$ is an arbitrary positive number less than $R$. The equation

implies then

$$
|r(z)-f(z)|=M, w \text { on } C,
$$

$$
\left|s(w)-\omega_{n}^{\prime}(w)\right| \leqq M+\frac{M^{\prime}}{R_{1}^{n}},
$$

for $s(w)=r(z)-f(z)+g(w)$. We may obviously write, then, by the definition of the Tchebycheff polynomials $\omega_{n}(w)$,

$$
\left|s(w)-\omega_{n}(w)\right| \leqq M+\frac{M^{\prime}}{R_{1}^{n}}
$$

we rewrite this inequality in the form

$$
\left|r(z)-\left[f(z)-g(w)+\omega_{n}(w)\right]\right| \leqq M+\frac{M^{\prime}}{R_{1}^{n}},
$$


which holds for $w$ in $B$ approaching $C$, and hence for $|z|<1,|z| \rightarrow 1$. This inequality implies, by (5.13),

for $|z|=\rho<1$, which is the same as

$$
\left|\left[f(z)-g(w)+\omega_{n}(w)\right]-f(z)\right| \leqq \frac{M^{\prime \prime}}{R_{1}{ }^{n}},
$$

$$
\left|g(w)-\omega_{n}(w)\right| \leqq \frac{M^{\prime \prime}}{R_{1}^{n}}
$$

for $w$ on an arbitrary Jordan curve $C^{\prime}$ interior to $C$, where of course $M^{\prime \prime}$ depends on $C^{\prime}$. The fact that this inequality holds for an arbitrary $R_{1}<R$ and an arbitrary curve $C^{\prime}$ interior to $C$ yields, by virtue of Theorem 4 , Theorem 12 as stated. For when the curve $C^{\prime}$ approaches the curve $C$ uniformly, then the curve $C_{R_{1}}^{\prime}$ (image in the $w$-plane of the circle $|z|=R_{1}>1$ if the exterior of $C^{\prime}$ is mapped onto the exterior of $|z|=1$ so that the points at infinity correspond to each other) approaches the curve $C_{R_{1}}$ uniformly. This follows from Carathéodory's results on conformal mapping.

The analogue of Theorem 10 will now be proved.

THEOREM 13. If in Theorem 11 the finite region $B$ is such that its boundary $C$ is also the boundary of an infinite region, then the sequence $\left\{\omega_{n}(w)\right\}$ of Tchebycheff polynomials for approximation on $C$ to the function $s(w)$ converges throughout $B$ to the function $g(w)$, uniformly on any closed point set interior to $B$.

We consider the sequence of analytic Jordan curves $C_{\mu}$ exterior to $B$ used in the proof of Theorem 10. The problem of approximation to $s(w)$ on $C_{\mu}$ will be studied in connection with the problem of approximation to $s(w)$ on $C$. Let $g^{(\mu)}(w)$ denote the function $g(w)$ of Theorem 11 which corresponds to the former problem, and $r^{(\mu)}(z)$ the rational function of $z$ which gives the equivalent problem of approximation in the unit circle in the z-plane:

$$
s(w)=r^{(\mu)}(z)-s_{1}^{(\mu)}(z), \quad s(w)-g^{(\mu)}(w)=r^{(\mu)}(z)-f^{(\mu)}(z),
$$

where $w=\phi_{\mu}(z)$, the function which maps $B_{\mu}$ onto $|z|<1$ and has auxiliary conditions independent of $\mu$.

The singularities of the function

$$
r(z)-f(z)=s(w)-g(w)
$$

(where $w=\phi(z)$, the function which maps $B$ onto $|z|<1$ and has the same auxiliary conditions as the $\left.\phi_{\mu}(z)\right)$ for $|z|<1$ differ but slightly from those of the function $r^{(\mu)}(z)-f^{(\mu)}(z)$. For these singularities depend merely on the singularities of $s(w)$ and on the values of the mapping functions and their derivatives in the neighborhoods of those singularities. The singularities vary continuously with the mapping functions (that is, the coefficients of 
the Laurent series for development about the singularities vary continuously) and so does $r^{(\mu)}(z)-f^{(\mu)}(z)$, by $\$ 3$ and equation (4.6). In fact we have

$$
\lim _{\mu \rightarrow \infty}\left[r^{(\mu)}(z)-f^{(\mu)}(z)\right]=r(z)-f(z)
$$

uniformly for $|z| \leqq 1$, except in the neighborhoods of the singularities of $r(z)$, for it is true that $\lim _{\mu \rightarrow \infty} \phi_{\mu}(z)=\phi(z)$ uniformly for $|z| \leqq \rho<1$.

Let an arbitrary positive $\epsilon$ be given. Choose $\mu$ so large that we have

$$
M^{(\mu)}<M+\frac{\epsilon}{3},
$$

where $M^{(\mu)}=\left|r^{(\mu)}(z)-f^{(\mu)}(z)\right|$ for $|z|=1$. Choose $\mu$ so large that we have also

$$
\left|s(w)-g^{(\mu)}(w)\right|<M^{(\mu)}+\frac{\epsilon}{3}
$$

for $w$ on $C$ and hence for $w$ in some neighborhood of $C$. This choice is possible, for we have as $\mu$ becomes infinite

$$
r^{(\mu)}(z)-f^{(\mu)}(z)=s(w)-g^{(\mu)}(w) \rightarrow r(z)-f(z)
$$

uniformly in the neighborhood of the circle $|z|=1$ (indeed throughout $|z| \leqq 1$ except in the neighborhood of the singularities of $r(z)$ ), where the second member of the equality is transformed by $w=\phi_{\mu}(z)$. Under the transformation, it is to be noticed, the point set $C$ corresponds to a closed point set in the neighborhood of $|z|=1$, and this closed point set approaches $|z|=1$ uniformly when $\mu$ becomes infinite.

It is now possible, by Theorem 12 , to choose the polynomial $\tau(w)$ so that for a particular $\mu$ (but sufficiently large to ensure the two inequalities just written) we have

$$
\left|g^{(\mu)}(w)-\tau(w)\right|<\frac{\epsilon}{3}
$$

for $w$ in some neighborhood of $C$; in fact this inequality can be satisfied on and within $C_{\mu}$. That is to say, we have

$$
|s(w)-\tau(w)|<M+\epsilon
$$

for $w$ in the neighborhood of $C$.

Inequality (8.7) informs us that there exists some polynomial $\tau(w)$ such that the left-hand member is less than $M+\epsilon$. It follows that the monotonically decreasing sequence

$$
\max \left|s(w)-\omega_{n}(w)\right|, \text { for } w \text { on } C,
$$


which approaches a limit, can approach no limit greater than $M$. This sequence can obviously, by Theorem 11, approach no limit less than $M$. Hence the limit of the sequence is $M$, and Theorem 13 is a consequence of Theorem 11.

The general problem of Theorem 11 is especially interesting if $s(w)$ is of the form $a / w$, where $a$ is a constant not zero. Under the transformation $w=\phi(z)$ we find that the function $r(z)$ of $(8.3)$ is of the form $b / z$, if $\phi(0)=0$, so we refer to the problem of Theorem 3 where $r(z)=b / z, b \neq 0$. In this case we have $f(z) \equiv 0$, so

$$
s(w)-g(w)=r(z)-f(z)=\frac{b}{z}
$$

is a function which maps $B$ onto the exterior of a circle of radius $|b|$ in the $z$-plane, in such a manner that the point $w=0$ corresponds to the point $z=\infty$.

9. Approximation to more general functions. Certain generalizations of the results previously obtained are entirely obvious. Let the function $r_{1}(z)$ have no singularities interior to $C:|z|=1$ other than a finite number of poles,

$$
r_{1}(z)=r(z)+r_{2}(z)
$$

where $r(z)$ is a rational function of $z$ with all of its singularities interior to $C$, and where $r_{2}(z)$ is analytic interior to $C$. Then the reader can easily prove (compare Theorem 3): Among all functions $f^{\prime}(z)$ analytic for $|z|<1$, there exists a unique function such that

$$
\underset{\substack{|s| \rightarrow 1 \\|z|<1}}{\operatorname{bound}}\left|r_{1}(z)-f^{\prime}(z)\right|
$$

is least; this function $f^{\prime}(z)$ can be written $f(z)+r_{2}(z)$, where $f(z)$ is rational. The function $r_{1}(z)-f^{\prime}(z)$ is of constant modulus $M$ on $C$. If the functions $f_{n}^{\prime}(z)$ are analytic for $|z|<1$, then

$$
\lim _{n \rightarrow \infty}\left[\prod_{\substack{b o u n d \\|z|<1}}\left|r_{1}(z)-f_{n}^{\prime}(z)\right|\right]=M
$$

implies $\lim _{n \rightarrow \infty} f_{n}^{\prime}(z)=f^{\prime}(z)$ for $|z|<1$, uniformly for $|z| \leqq \rho<1$.

Later theorems of $\$ 8$ likewise have analogues in this new situation; we sketch the modifications to be made. We assume (compare Theorem 11) $s_{1}(w)$ to have no singularities in $B$ other than a finite number of poles:

$$
s_{1}(w)=s(w)+s_{2}(w),
$$

where $s(w)$ is a rational function of $w$ with all of its singularities in $B$ and $s_{2}(w)$ is analytic in $B$. Of all functions $g^{\prime}(w)$ analytic interior to $B$, there exists a unique function such that

$$
\underset{\substack{w \rightarrow C \\ w \text { in } B}}{\operatorname{bound}}\left|s_{1}(w)-g^{\prime}(w)\right|
$$


is least; the function $s_{1}(w)-g^{\prime}(w)$ is of constant modulus $M$ on $C$-that is, the modulus of $s_{1}(w)-g^{\prime}(w)$ approaches a constant value $M$ as $w$ in $B$ approaches $C$. If the functions $g_{n}^{\prime}(w)$ are analytic for $w$ in $B$, then

$$
\lim _{n \rightarrow \infty}\left[\underset{\substack{w \rightarrow C \\ w \text { in } B}}{\operatorname{bound}}\left|s_{1}(w)-g_{n}^{\prime}(w)\right|\right]=M
$$

implies $\lim _{n \rightarrow \infty} g_{n}^{\prime}(w)=g^{\prime}(w)$ for $w$ in $B$, uniformly for $w$ on any closed point set in $B$.

Theorem 12 holds unchanged if $s(w)$ and $g(w)$ are replaced by $s^{\prime}(w)$ and $g^{\prime}(w)$ respectively. Theorem 13 holds also if, in our present notation, the function $s_{2}(w)$ can be uniformly approximated in $B+C$ as closely as desired by a polynomial in $w$, and thus holds in particular if $B$ is a Jordan region and $s_{2}(w)$ is analytic in $B$, continuous in $B+C$.

The situation of Theorem 1 is also easy to generalize. Let the function $\Phi(z)$ be given analytic interior to $C:|z|<1$; we consider the problem of determining the function $\phi(z)$ also analytic for $|z|<1$, which takes on prescribed values at preassigned points $\alpha_{1}, \alpha_{2}, \cdots, \alpha_{k}$ interior to $C$, and such that

$$
\overline{\text { bound }}|\Phi(z)-\phi(z)|, \text { for }|z|<1 \text {, }
$$

is least. Any function $F(z)$ analytic for $|z|<1$ which takes on the values $\Phi\left(\alpha_{i}\right)-\phi\left(\alpha_{i}\right)$ at the points $\alpha_{i}$ can be written in the form

$$
F(z)=\Phi(z)-\phi(z),
$$

where this equation defines $\phi(z)$. Conversely, if $\phi(z)$ is given, a corresponding function $F(z)$ analytic for $|z|<1$ and taking on the values $\Phi\left(\alpha_{i}\right)-\phi\left(\alpha_{i}\right)$ at the points $\alpha_{i}$ can be considered defined by (9.2). The entire problem of the existence, uniqueness, and determination of $\phi(z)$ is equivalent to the problem of the existence, uniqueness, and determination of the function $F(z)$ of Theorem 1 and can then be considered solved. The reader should have no difficulty in writing down the analogues of Theorems $1,2,7,8,9,10$.

Suppose the function $\Phi(z)$ is an arbitrary function of $z$ with not more than a finite number of poles and no other singularities interior to $C:|z|=1$; we consider as in Theorem B the problem of determining the function $\phi(z)$ analytic interior to $C$ which takes on prescribed values at preassigned points $\alpha_{1}, \alpha_{2}, \cdots, \alpha_{k}$ interior to $C$ and is such that

$$
\underset{\substack{|z| \rightarrow 1 \\|z|<1}}{\mid}|\Phi(z)-\phi(z)|
$$

is least. Let $\beta_{1}, \beta_{2}, \cdots, \beta_{l}$ denote the poles of $\Phi(z)$ interior to $C$, each pole 
enumerated the number of times corresponding to its multiplicity; then (9.3) is precisely the same as

$$
\underset{\substack{\text { bound } \\|z| \rightarrow 1 \\|z|<1}}{ }\left|[\Phi(z)-\phi(z)] \frac{\left(z-\beta_{1}\right)\left(z-\beta_{z}\right) \cdots\left(z-\beta_{l}\right)}{\left(1-\bar{\beta}_{1} z\right)\left(1-\bar{\beta}_{2} z\right) \cdots\left(1-\bar{\beta}_{l} z\right)}\right| .
$$

This last expression shows that our problem is equivalent to the problem of approximating the function

$$
\Phi_{1}(z)=\Phi(z) \frac{\left(z-\beta_{1}\right)\left(z-\beta_{2}\right) \cdots\left(z-\beta_{\imath}\right)}{\left(1-\bar{\beta}_{1} z\right)\left(1-\bar{\beta}_{2} z\right) \cdots\left(1-\bar{\beta}_{l} z\right)},
$$

which is analytic interior to $C$, by the function

$$
\phi_{1}(z)=\phi(z) \frac{\left(z-\beta_{1}\right)\left(z-\beta_{2}\right) \cdots\left(z-\beta_{l}\right)}{\left(1-\bar{\beta}_{1} z\right)\left(1-\bar{\beta}_{2} z\right) \cdots\left(1-\bar{\beta}_{l} z\right)},
$$

also analytic interior to $C$, a problem of the type just considered. Determination of the function $\phi(z)$ with the prescribed auxiliary conditions leads to the determination of $\phi_{1}(z)$ with the conditions

$$
\begin{aligned}
& \phi_{1}\left(\alpha_{i}\right)=\phi\left(\alpha_{i}\right) \frac{\left(\alpha_{i}-\beta_{1}\right)\left(\alpha_{i}-\beta_{2}\right) \cdots\left(\alpha_{i}-\beta_{l}\right)}{\left(1-\bar{\beta}_{1} \alpha_{i}\right)\left(1-\bar{\beta}_{2} \alpha_{i}\right) \cdots\left(1-\bar{\beta}_{l} \alpha_{i}\right)} \quad(i=1,2, \cdots, k), \\
& \phi_{1}\left(\beta_{i}\right)=0 \quad\left(i=1,2, \cdots, l, \quad \beta_{i} \neq \alpha_{i}\right) ;
\end{aligned}
$$

and reciprocally, determination of $\phi_{1}(z)$ with these auxiliary conditions leads to the determination of $\phi(z)$ satisfying the prescribed conditions. Obvious alterations in these conditions are here necessary if the $\beta_{i}$ are not all distinct, or if derivatives of $\phi(z)$ are prescribed as well as functional values. The usual conventions suffice, however, even if some of the $\alpha_{i}$ coincide with some of the $\beta_{i}$.

We add a remark on the degrees of the functions involved. The function $F(z)=\Phi_{1}(z)-\phi_{1}(z)$ is the minimizing function (as in Theorem 1) corresponding to $k+l$ auxiliary conditions and hence is of degree less than $k+l$. We have

$$
\Phi(z)-\phi(z)=\frac{\left(1-\bar{\beta}_{1} z\right)\left(1-\bar{\beta}_{2} z\right) \cdots\left(1-\bar{\beta}_{l} z\right)}{\left(z-\beta_{1}\right)\left(z-\beta_{2}\right) \cdots\left(z-\beta_{l}\right)} F(z),
$$

which is therefore of degree less than $k+2 l$.

We state the result as a theorem not in the z-plane but after conformal mapping onto the w-plane; the detailed reasoning involved is already familiar to the reader.

THEOREM 14. Let $B$ be an arbitrary simply connected region of the w-plane, with the boundary $C$. Let $\Psi(w)$ be analytic in $B$ except possibly for a finite number of poles. Let $\psi(w)$ denote generically a function analytic in $B$ which at 
$k$ given points $P$ of $B$ has preassigned values for itself and its first $k_{P}$ derivatives. Then a unique function $\psi(w)$ exists so that

$$
\underset{\substack{w \rightarrow C \\ w \text { in } B}}{\operatorname{cound}}|\Psi(w)-\psi(w)|
$$

is least. The modulus of $\Psi(w)-\psi(w)$ approaches a constant value $M$ as $w$ remaining in $B$ approaches $C$.

If the functions $\psi_{n}(w)$ analytic in $B$ satisfy the auxiliary conditions, then

$$
\lim _{n \rightarrow \infty}\left[\underset{\substack{w \rightarrow C \\ w \text { in } B}}{\operatorname{bound}}\left|\Psi(w)-\psi_{n}(w)\right|\right]=M
$$

implies

$$
\lim _{n \rightarrow \infty} \psi_{n}(w)=\psi(w)
$$

for w in $B$, uniformly on any closed point set interior to $B$.

Theorem 14 can be interpreted as a direct extension of Theorem 1 , where not only finite but also infinite values of the function may be assigned at the $k^{\prime}$ points $P$ (i.e., analogous to the points $P$ of Theorem 8 ) interior to $B$. These $k^{\prime}$ points $P$ are of course the $k$ points $P$ of Theorem 14 plus the poles of $\Psi(w)$ interior to $B$. That is to say, we consider the class of functions $\Psi(w)-\psi(w)$ of the form

$$
\begin{aligned}
a_{-q}(w-p)^{-q}+a_{-q+1}(w-p)^{-q+1} & +\cdots+a_{0}+a_{1}(w-p)+\cdots \\
& +a_{m}(w-p)^{m}+b_{m+1}(w-p)^{m+1}+\cdots,
\end{aligned}
$$

where at each of the $k^{\prime}$ given points $P: w=p$, the numbers $a_{i}$ (depending on $P$ ) are prescribed and the numbers $b_{i}$ are not prescribed; the restriction $m>0$ or $m \geqq 0$ is not made. We naturally consider as a measure of the modulus of $F(z)=\Psi(w)-\psi(w)$ not the upper limit in $B$ of the modulus of the function, but as in Theorem 11,

$$
\underset{\substack{w \rightarrow C \\ w \text { in } B}}{\operatorname{bound}}|\Psi(w)-\psi(w)| \text {. }
$$

There is no difference in these measures of the modulus of $F(z)$ if $F(z)$ is analytic interior to $B$ (mapped on the unit circle in the $z$-plane) but of course there is a difference if the auxiliary conditions involve poles of $F(z)$. There exists a unique function $F(z)=\Psi(w)-\psi(w)$ of this class whose least upper bound for $w$ in $B$ approaching $C$ is less than the least upper bound for $w$ in $B$ approaching $C$ for any other function of the class. This function is rational in $z$ (that is, when $B$ is mapped on the unit circle in the z-plane) and of constant modulus $M$ on $C$, in the sense that

$$
\lim _{\substack{w \rightarrow C \\ w \text { in } B}}|\Psi(w)-\psi(w)|=M .
$$


An arbitrary rational function $r(z)$ of constant modulus on $\gamma:|z|=1$ can be written in the form

$$
r(z)=K \frac{\left(z-\alpha_{1}\right)\left(z-\alpha_{2}\right) \cdots\left(z-\alpha_{\mu}\right)\left(1-\bar{\beta}_{1} z\right)\left(1-\bar{\beta}_{2} z\right) \cdots\left(1-\bar{\beta}_{\nu} z\right)}{\left(1-\bar{\alpha}_{1} z\right)\left(1-\bar{\alpha}_{2} z\right) \cdots\left(1-\bar{\alpha}_{\mu} z\right)\left(z-\beta_{1}\right)\left(z-\beta_{2}\right) \cdots\left(z-\beta_{v}\right)} .
$$

Let $r(z)$ not be identically zero and let $\alpha_{1}, \alpha_{2}, \cdots, \alpha_{\mu}$ denote the zeros of $r(z)$ interior to $\gamma$ and $\beta_{1}, \beta_{2}, \cdots, \beta_{v}$ the poles of $r(z)$ interior to $\gamma$. Then the function

$$
r(z) \frac{\left(1-\bar{\alpha}_{1} z\right) \cdots\left(1-\bar{\alpha}_{\mu} z\right)\left(z-\beta_{1}\right) \cdots\left(z-\beta_{\nu}\right)}{\left(z-\alpha_{1}\right) \cdots\left(z-\alpha_{\mu}\right)\left(1-\bar{\beta}_{1} z\right) \cdots\left(1-\bar{\beta}_{\nu} z\right)}
$$

is analytic and different from zero on and within $\gamma$ and of constant modulus on $\gamma$, hence is a constant.

It is perhaps worth while to give the details of the proof of the following addition to Theorem 14:

TheOREM 15. Suppose $B$ is a finite region whose boundary $C$ is also the boundary of an infinite region; and suppose when we write

$$
\Psi(w)=\Psi_{1}(w)+\Psi_{2}(w),
$$

where $\Psi_{1}(w)$ is a rational function of $w$ and has in $B$ precisely the singularities of $\Psi(w)$ and is analytic on and exterior to $C$, the function $\Psi_{2}(w)$ can be uniformly approximated on $B+C$ as closely as desired by a polynomial in w; then the sequence $\left\{\omega_{n}(w)\right\}$ of Tchebycheff polynomials for approximation on $C$ to the function $\Psi(w)$ with arbitrary given auxiliary conditions, converges to the function $\psi(w)$ of Theorem 14 throughout $B$, uniformly on any closed point set interior to $B$.

If moreover $C$ is an analytic Jordan curve, and if the function $\psi(w)$ is analytic on and interior to $C_{R}$, then the sequence $\left\{\omega_{n}(w)\right\}$ converges uniformly on and within $C_{R}$.

We prove first the second part of Theorem 15. We have by Theorem 4 the existence of a sequence of polynomials $\omega_{n}^{\prime}(w)$ of respective degrees $n$ such that

$$
\left|\psi(w)-\omega_{n}^{\prime}(w)\right| \leqq \frac{M^{\prime}}{R^{n}} \text { for } w \text { on } B+C .
$$

These polynomials $\omega_{n}^{\prime}(w)$ are chosen also to satisfy the given auxiliary conditions; the function $\psi(w)$ satisfies those conditions and hence (as in the proof of Theorem 7) the polynomials $\omega_{n}^{\prime}(w)$ can likewise be chosen to satisfy them. We have also

$$
\lim _{\substack{w \rightarrow C \\ w \text { in } B}}|\Psi(w)-\psi(w)|=M,
$$


and thus derive

$$
\left|\Psi(w)-\omega_{n}^{\prime}(w)\right| \leqq M+\frac{M^{\prime}}{R^{n}}, \text { for } w \text { on } C .
$$

It follows by the definition of the Tchebycheff polynomial $\omega_{n}(w)$ that we have

$$
\left|\Psi(w)-\omega_{n}(w)\right| \leqq M+\frac{M^{\prime}}{R^{n}}, \text { for } w \text { on } C ;
$$

the result as stated in the theorem now follows as in the proof of Theorem 12. It will be noticed that by (9.4) an inequality of type (5.13) holds indifferently for approximation to functions of type $\Phi(z)$ as used in (9.3) by a sequence of functions of type $\phi(z)$, or for approximation to functions of type $\Phi_{1}(z)$ by a sequence of functions of type $\phi_{1}(z)$.

We turn now to the proof of the first part of Theorem 15. Consider new auxiliary conditions, so that the prescribed values for the new polynomial $\omega^{\prime \prime}(w)$ now to be introduced differ from those of $\omega_{n}(w)$ by the values of $\Psi_{2}(w)$ in the respective points:

$$
\omega^{\prime \prime}(w)+\Psi_{2}(w)=\omega_{n}(w) \text {, when } w \text { is a point } P .
$$

It follows from the proof of Theorem 13 by the use of the reasoning used in the proof of Theorem 7 , that a polynomial $\omega^{\prime \prime}(w)$ of some degree $m$ exists which satisfies the new auxiliary conditions and is such that we have

$$
\left|\left[\Psi(w)-\Psi_{2}(w)\right]-\omega^{\prime \prime}(w)\right| \leqq M+\frac{\epsilon}{2}, \text { for } w \text { on } C,
$$

where $\epsilon$ is arbitrary but preassigned. For it is obvious that if in Theorem 14 the function $\Psi(w)$ is replaced by $\Psi_{1}(w)=\Psi(w)-\Psi_{2}(w)$, and the auxiliary conditions satisfied by $\psi(w)$ are replaced by the values of $\psi(w)-\Psi_{2}(w)$ at the points $P$, then $\psi(w)$ is replaced by $\psi(w)-\Psi_{2}(w)$ and $M$ is unchanged.

There exists by the hypothesis on $\Psi_{2}(w)$ and by the use of an auxiliary theorem, ${ }^{*}$ a polynomial $\omega(w)$ which takes on the same values as $\Psi_{2}(w)$ in the points $P$ and is such that

$$
\left|\Psi_{2}(w)-\omega(w)\right| \leqq \frac{\epsilon}{2}, \text { for } w \text { on } B+C .
$$

There results by means of (9.5) the inequality

$$
\left|\Psi(w)-\left[\omega(w)+\omega^{\prime \prime}(w)\right]\right| \leqq M+\epsilon, \text { for } w \text { on } C,
$$

\footnotetext{
* Walsh, these Transactions, vol. 30 (1928), pp. 307-332; Theorem X, p. 319. See also the proof of Theorem 7 of the present paper.
} 
and the polynomial $\omega(w)+\omega^{\prime \prime}(w)$ satisfies the given auxiliary conditions for the $\omega_{n}(w)$. That is, the greatest lower bound of

$$
|\Psi(w)-\pi(w)|, \text { for } w \text { on } C,
$$

for all polynomials $\pi(w)$ satisfying the auxiliary conditions, is not greater than $M$, so we have

$$
\lim _{n \rightarrow \infty}\left[\text { bound }\left|\Psi(w)-\omega_{n}(w)\right|, w \text { on } C\right]=M,
$$

and the desired result is a consequence of Theorem 14.

We mention explicitly that if an arbitrary function $\Psi_{2}(w)$ has no singularities other than in the infinite region of which $C$ is the boundary, then on $B+C$ the function $\Psi_{2}(w)$ can be uniformly approximated as closely as desired by a polynomial in $w^{*}$ and hence satisfies the conditions of Theorem 15 .

It would be interesting to study the approximation on $C$ by polynomials of a function with singularities other than in $B$ and in the infinite region of which $C$ is the boundary; this problem arises only when $C$ separates the plane into more than two regions, and for the present the problem must remain unsolved.

There are evidently other questions that are left unanswered by the present treatment. Is it true ${ }^{2}$ that if the region $B$ in Theorem 15 is a Jordan region the sequence $\left\{\omega_{n}(w)\right\}$ converges uniformly to the function $\psi(w)$ in the closed region? What can be said if in Theorem 15 the function $\Psi(w)$ is simply an arbitrary function continuous on $C$ ? What are the facts if in Theorem 14 we do not require the number of points $P$ to be finite? Under suitable restrictions much of the reasoning we have given can be extended with ease-for instance the proof of the existence of the minimizing function in Theorem 1 holds for approximation on $C$ to an arbitrary limited function (continuous or not) defined on $C$-but under other conditions it may happen (compare Nevanlinna, loc. cit., Abschnitt II) that the discussion given requires substantial modification.

10. Existence and uniqueness of the Tchebycheff polynomial with auxiliary conditions. For the situation of Theorem 15, the existence of the Tchebycheff polynomial with auxiliary conditions follows (in case the degree of the polynomial is so large that it is possible to make the polynomial satisfy the auxiliary conditions) from Montel's theory of normal families of functions. For the class of all polynomials $\omega_{n}(w)$ of a given degree $n$ satisfying the auxiliary conditions, the number

*Walsh, Mathematische Annalen, vol. 96 (1926), pp. 437-450; footnote, p. 441.

$\dagger$ The corresponding question is raised by Julia (loc.cit.) for the special problem treated by him, but is not answered. 


$$
\omega_{n}=\max \left|\Psi(w)-\omega_{n}(w)\right|, \text { for } w \text { on } C,
$$

has a greatest lower bound $b$. There exists a sequence of polynomials $\omega_{n}{ }^{(k)}(w)$ all of degree $n$ such that

$$
\lim _{k \rightarrow \infty}\left[\max \left|\Psi(w)-\omega_{n}^{(k)}(w)\right|, \text { for } w \text { on } C\right]=b .
$$

From this sequence can be extracted a subsequence which converges in $B$, uniformly on an arbitrary point set interior to $B$. The limit of this subsequence is then a polynomial $\omega_{n}(w)$ of degree $n$ which satisfies the auxiliary conditions, the convergence is uniform in $B+C$, and we have for this particular polynomial

$$
b=\max \left|\Psi(w)-\omega_{n}(w)\right|, \text { for } w \text { on } C .
$$

The existence of the Tchebycheff polynomial with auxiliary conditions can, however, be established under much more general conditions.* We do not dwell on this point, but turn to the proof of the uniqueness of the Tchebycheff polynomial.

It is worth remarking that the proofs we have given for the convergence properties of the sequence of Tchebycheff polynomials do not depend in any way on the uniqueness of those polynomials, so that the material about to be given is not logically necessary by way of justification.

Let $f(z)$ be an arbitrary function of $z$ continuous on a closed limited point set $C$ containing at least $n+2$ points. $\dagger$ Then the Tchebycheff polynomial $\pi_{n}(z)$ of degree $n$ for approximation to $f(z)$ on $C$ with the auxiliary conditions

$$
\pi_{n}\left(\alpha_{i}\right)=\gamma_{i} \quad(i=1,2, \cdots, k)
$$

is unique provided $n \geqq k-1$ and provided that $\gamma_{i}=f\left(\alpha_{i}\right)$ if $\alpha_{i}$ is a point of $C$.

Special cases occur here if some of the points $\alpha_{i}$ coincide with each other, so that (10.1) is considered to restrict not merely $\pi_{n}\left(\alpha_{i}\right)$ but also one or more derivatives of the polynomial for the value $z=\alpha_{i}$; we use $k$ to denote the total number of equations (10.1), not the number of distinct points $\alpha_{i}$. It is not necessary for the truth of this theorem so to restrict the values of these derivatives of the Tchebycheff polynomials, if any are prescribed, that they coincide with the derivatives (if any exist) of $f(z)$, even if $\alpha_{i}$ is a point of $C$.

The proof which follows is, except for minor changes due to the introduction of the auxiliary conditions (10.1), precisely the proof given by Tonelli for the case $k=0 . \ddagger$ For that reason, we give the proof merely in outline and

* By the methods used by de la Vallée Poussin, Approximation des Fonctions, Paris, 1919, p. 75. $\dagger$ The proof holds, with only minor modifications, if we have but $n+1$ points in $C$.

$\ddagger$ Annali di Matematica, (3), vol. 15 (1908), pp. 108-113. 
refer to Tonelli for the details.*

Let $\pi_{n}(z)$ denote a Tchebycheff polynomial of degree $n$, and consider the difference

$$
f(z)-\pi_{n}(z)=Y(z),
$$

which is likewise continuous on $C$ and has on $C$ a maximum modulus $\mu$. We shall prove first that the number of distinct points of $C$ in which $Y(z)$ reaches its maximum absolute value is greater than $n-k+1$.

We suppose the contrary, that $|Y(z)|$ reaches its maximum absolute value in the points $z_{1}, z_{2}, \cdots, z_{\nu}$ of $C$, where $\nu \leqq n-k+1$. It will be noticed that no point $z_{i}$ coincides with an $\alpha_{j}$. Let $P(z)$ be a polynomial of degree $n$ (i.e. in the sense of $\$ 5$ ) such that

$$
\begin{array}{ll}
P\left(z_{i}\right)=Y\left(z_{i}\right) & (i=1,2, \cdots, \nu), \\
P\left(\alpha_{i}\right)=0 & (i=1,2, \cdots, k),
\end{array}
$$

where the second equation is to be interpreted as requiring the suitable derivatives of $P(z)$ to vanish if the $\alpha_{i}$ are not all distinct. Such a polynomial $P(z)$ of degree $n$ always exists.

Let an arbitrary positive $\epsilon<\mu / 2$ be given. By the continuity of the functions $Y(z)$ and $P(z)$ on $C$, there exists a $\delta$ so that $\left|z^{\prime}-z^{\prime \prime}\right|<\delta$ implies

$$
\left|Y\left(z^{\prime}\right)-Y\left(z^{\prime \prime}\right)\right|<\epsilon, \quad\left|P\left(z^{\prime}\right)-P\left(z^{\prime \prime}\right)\right|<\epsilon .
$$

Denote by $C^{\prime}$ the point set common to $C$ and the circles

$$
\left|z-z_{i}\right|<\delta,
$$

and by $C^{\prime \prime}$ the complementary set with respect to $C$. On $C^{\prime \prime}$ the function $Y(z)$ has a maximum absolute value $\mu^{\prime \prime}<\mu$ :

$$
|Y(z)| \leqq \mu^{\prime \prime} .
$$

On $C$, the polynomial $P(z)$ has some maximum modulus $M$.

If the positive number $\omega$ is chosen less than $\left(\mu-\mu^{\prime \prime}\right) /(2 M)$, we have

$$
|\omega P(z)|<\left(\mu-\mu^{\prime \prime}\right) / 2
$$

for $z$ on $C$. If $\omega$ is chosen sufficiently small, still less than $\left(\mu-\mu^{\prime \prime}\right) /(2 M)$, we have (Tonelli, loc. cit., pp. 110-111)

$$
|Y(z)-\omega P(z)|<\mu^{\prime}<\mu
$$

for $z$ on $C^{\prime}$; this inequality is proved first for each individual circle (10.2), where $\mu^{\prime}$ depends on the circle, and hence follows for the entire point set $C^{\prime}$.

* The present writer takes exception to the corresponding discussion given by Julia, in particular to the statement (loc. cit., pp. 291-292) "en tout point où $\left|\pi_{n}\right|$ atteint son maximum, $\left|Q_{n}\right|$ atteint aussi son maximum." The fact is of course true, but one cannot conclude a priori without further demonstration that $\left|Q_{n}\right|$ attains its maximum in more than a single point in which $\left|\pi_{n}\right|$ attains its maximum. 
But we have for $z$ on $C^{\prime \prime}$

$$
\begin{aligned}
\left|f(z)-\left[\pi_{n}(z)+\omega P(z)\right]\right| & =|Y(z)-\omega P(z)| \leqq|Y(z)|+|\omega P(z)| \\
& \leqq \mu^{\prime \prime}+\frac{\mu-\mu^{\prime \prime}}{2}=\frac{\mu+\mu^{\prime \prime}}{2}<\mu .
\end{aligned}
$$

We have thus exhibited a new polynomial $\pi_{n}(z)+\omega P(z)$ of degree $n$ which satisfies the auxiliary conditions and which by (10.3) and (10.4) deviates from $f(z)$ on $C$ by a quantity in absolute value less than $\mu$, which contradicts the assumption that $\pi_{n}(z)$ is a Tchebycheff polynomial.

The uniqueness of the Tchebycheff polynomial will now be established from the fact just proved, that the difference $Y(z)$ reaches its maximum absolute value in more than $n-k+1$ points. Assume two Tchebycheff polynomials of degree $n$ to exist, $\pi_{n}(z)$ and $\pi_{n}^{\prime}(z)$, and let $\mu$ have the same significance as before. Then we have for $z$ on $C$

$$
\left|f(z)-\frac{\pi_{n}(z)+\pi_{n}^{\prime}(z)}{2}\right| \leqq \frac{\left|f(z)-\pi_{n}(z)\right|}{2}+\frac{\left|f(z)-\pi_{n}^{\prime}(z)\right|}{2} \leqq \mu
$$

so that $\left[\pi_{n}(z)+\pi_{n}^{\prime}(z)\right] / 2$ is likewise a Tchebycheff polynomial of degree $n$ which satisfies the given auxiliary conditions. The left-hand member of (10.5) can equal $\mu$ for a particular value of $z$ only if both of the two terms in the next member have the value $\mu / 2$, and indeed only if the two expressions

$$
f(z)-\pi_{n}(z), f(z)-\pi_{n}^{\prime}(z)
$$

are equal for this particular value of $z$. There are, as we have just shown, at least $n-k+2$ distinct values of $z$ (necessarily distinct from the $\alpha_{i}$ ) at which the left-hand member of (10.5) has the value $\mu$, so there are at least $n-k+2$ distinct values of $z$ different from the $\alpha_{i}$ at which $\pi_{n}(z)$ and $\pi_{n}^{\prime}(z)$ are equal. The difference of these two polynomials has at least $n+2$ common roots, if possible multiple roots at the $\alpha_{i}$ are counted according to their multiplicities, so this difference vanishes identically, the polynomials $\pi_{n}(z)$ and $\pi_{n}^{\prime}(z)$ are identical, and the theorem is completely established.

The simplest possible examples show that the theorem fails if there is omitted the restriction $\gamma_{i}=f\left(\alpha_{i}\right)$ when $\alpha_{i}$ is a point of $C$.

11. Convergence and divergence of approximating sequence on the boundary. We are not in a position to give a complete discussion of the convergence on the circumference $C$ of the sequence $\left\{F_{n}(z)\right\}$ of Theorem 2, but we shall give here certain positive and negative results concerning this convergence. We have already given $(\$ 4)$ an example to show that $\lim _{n \rightarrow \infty}$ $F_{n}(z)=F(z)$ may fail at a single point of $C$, even when the functions $F_{n}(z)$ 
are analytic on as well as within $C$. At exceptional points it may occur that we have $\lim _{n \rightarrow \infty} F_{n}(z)=0$. Indeed, if we choose

$$
F_{n}(z)=e^{n /\left[n^{2}(z-1)-1\right]+n /\left(n^{2}+1\right)},
$$

we have fulfilled the requirements (in particular $\left.F_{n}(0)=1\right)$ of the functions considered in $\$ 4$ with reference to Theorem 2 , and in addition we have

$$
\lim _{n \rightarrow \infty} F_{n}(z)=0 \text { for } z=1 .
$$

We shall now give an example to show that in Theorem 2 the equation $\lim _{n \rightarrow \infty} F_{n}(z)=F(z)$ may fail at every point of $C$. We make use of the function considered in $\S 4$ :

$$
\Phi_{n}(z)=e^{1 /[n(z-1)-1]+1 /(n+1)},
$$

so that we have $\Phi_{n}(1)=e^{-n /(n+1)}<e^{-1 / 2}, n>1 ; \Phi_{n}(-1)=e^{n /[(2 n+1)(n+1)]}>1$. There exists a positive quantity $\theta_{n}$ such that for $|\theta|<\theta_{n}$ we have

$$
\left|\Phi_{n}\left(e^{i \theta}\right)\right|<e^{-1 / 2},
$$

and for $|\theta-\pi|<\theta_{n}$ we have

$$
\left|\Phi_{n}\left(e^{i \theta}\right)\right|>1 .
$$

Choose $N_{n}$ such that $1 / N_{n}<\theta_{n} /(2 \pi)$. We shall use an $N_{n}$ th root of unity:

$$
\omega_{n}=e^{2 \pi i / N_{n}} \text {. }
$$

Consider the sequence $\left\{F_{n}(z)\right\}$ :

$$
\begin{aligned}
& \Phi_{2}\left(\omega_{2} z\right), \Phi_{2}\left(\omega_{2}^{2} z\right), \cdots, \Phi_{2}\left(\omega_{2}^{N_{2} z}\right), \\
& \Phi_{3}\left(\omega_{3} z\right), \Phi_{3}\left(\omega_{3}^{2} z\right), \cdots, \Phi_{3}\left(\omega_{3}^{N_{3} z}\right),
\end{aligned}
$$

where the rows of functions $\Phi_{n}\left(\omega_{n}^{i} z\right)$ are taken in order. The conditions of Theorem 2 (in particular $F_{n}(0)=1$ ) are satisfied, the functions $F_{n}(z)$ are analytic even for $|z|=1$, yet $\lim _{n \rightarrow \infty} F_{n}(z)$ exists for no value of $z$ on $C$. Indeed if an arbitrary $z=z_{0}$ on $C$ is chosen and an arbitrary $N$, then there exist $N^{\prime}$ and $N^{\prime \prime}$ such that

$$
\begin{array}{ll}
\left|F_{N^{\prime}}\left(z_{0}\right)\right|<e^{-1 / 2}, & N^{\prime}>N, \\
\left|F_{N}\left(z_{0}\right)\right|>1, & N^{\prime \prime}>N .
\end{array}
$$

We shall find it convenient to introduce some new terminology. Given the function $f(z)$ and the sequence of functions $\left\{f_{n}(z)\right\}$ defined on a certain point set $E$. Suppose that when an arbitrary $\epsilon>0$ is given there exists $N$ such that

$$
\left|f_{n}(z)-f(z)\right|<\epsilon, n \geqq N,
$$


except perhaps on a point set $E_{n}$ of measure less than $\epsilon$. Then we shall say that the sequence $\left\{f_{n}(z)\right\}$ is quasiconvergent or quasiconverges to the function $f(z)$ on the given point set, and we write

$$
\operatorname{qlim}_{n \rightarrow \infty} f_{n}(z)=f(z) \text {. }
$$

Actual convergence need not take place-in fact it will appear later that the sequence of functions $F_{n}(z)$ defined above in terms of the functions $\Phi_{n}\left(\omega_{n}^{i} z\right)$ quasiconverges on the circumference $C$ to the function unity.

The notion just introduced is related to Weyl's essentially uniform convergence. The sequence $\phi_{n}(z)$ converges essentially uniformly on $E$ to the function $\phi(z)$ if, when an arbitrary $\epsilon>0$ is given, the sequence $\phi_{n}(z)$ converges uniformly on $E$ to $\phi(z)$ except perhaps on a point set of measure less than $\epsilon$.

If the sequence $\left\{f_{n}(z)\right\}$ quasiconverges on $E$ to the function $f(z)$, then from every subsequence of the $\left\{f_{n}(z)\right\}$ can be extracted a new subsequence which converges on $E$ essentially uniformly to $f(z)$, and conversely, if from every subsequence of the functions $\left\{f_{n}(z)\right\}$ can be extracted a new subsequence which converges on $E$ essentially uniformly to $f(z)$, then the original sequence quasiconverges on $E$ to the function $f(z)$.

We prove first the direct theorem. Let $\epsilon>0$ be given and let $N_{1}, N_{2}, \cdots$ be the numbers in the definition of quasiconvergence corresponding to the values $\epsilon / 2, \epsilon / 4, \epsilon / 8, \cdots$. Let $f_{M_{k}}(z), M_{k} \geqq N_{k}$, be a function of the given subsequence. Then the new subsequence is to be $\left\{f_{M_{k}}(z)\right\}$ and we have

$$
\begin{aligned}
& \left|f_{M_{1}}(z)-f(z)\right|<\frac{\epsilon}{2}, \text { except perhaps on a set } E_{1} \text { of measure }<\frac{\epsilon}{2}, \\
& \left|f_{M_{2}}(z)-f(z)\right|<\frac{\epsilon}{4}, \text { except perhaps on a set } E_{2} \text { of measure }<\frac{\epsilon}{4},
\end{aligned}
$$

Thus the sequence $\left\{f_{M_{k}}(z)\right\}$ converges on $E$ uniformly to the function $f(z)$ except perhaps on the point set $E_{n+1}+E_{n+2}+\cdots$, whose measure is less than $\epsilon / 2^{n}$.

Let us prove now the converse theorem. If the theorem is not true, there exists an $\epsilon>0$ and an infinity of indices $n_{1}, n_{2}, \cdots$, such that

$$
\left|f_{n_{k}}(z)-f(z)\right| \geqq \epsilon
$$

on a point set of measure not less than $\epsilon$. Then from the sequence $\left\{f_{n_{k}}(z)\right\}$ can be extracted no subsequence which converges on $E$ essentially uniformly to $f(z)$, which contradicts our hypothesis.

A theorem due to Egoroff* is of interest here.

\footnotetext{
* Paris Comptes Rendus, vol. 152 (1911), p. 244.
} 
If $E$ be a measurable set of finite measure, a necessary and sufficient condition that a sequence $\left\{f_{n}(z)\right\}$ of measurable functions should converge to a function $f(z)$ almost everywhere on $E$ is that the sequence $\left\{f_{n}(z)\right\}$ converge essentially uniformly to $f(z)$ on $E$.

In the present paper we are concerned only with measurable functions. We combine Egoroff's theorem with the preceding one:

If $E$ be a measurable set of finite measure, a necessary and sufficient condition that a sequence $\left\{f_{n}(z)\right\}$ of measurable functions should quasiconverge to $f(z)$ on $E$ is that from every subsequence of the $\left\{f_{n}(z)\right\}$ can be extracted a new subsequence which converges almost everywhere to $f(z)$ on $E$.

We use the concept of quasiconvergence in a preliminary theorem:

Let the single auxiliary condition of Theorem 2 be $F_{n}(0)=1$. Then we have for the sequence $\left\{F_{n}(z)\right\}$ of Theorem 2 in the sense of quasiconvergence on the circumference $C$,

$$
\operatorname{qlim}_{n \rightarrow \infty} F_{n}(z)=1
$$

Each function $F_{n}(z)$ is, at least for $n$ sufficiently large, uniformly bounded interior to $C$. It follows from a well known theorem due to Fatou that

$$
\lim _{\rho \rightarrow 1} F_{n}\left(\rho e^{i \phi}\right), z=\rho e^{i \phi} \text {, }
$$

exists for almost all values of $\phi$. These values are the values of $F_{n}(z)$ to which we refer in the theorem to be proved.

If $C^{\prime}$ is the circle $|z|=\rho<1$, then we have Cauchy's integral

$$
F_{n}(0)=1=\frac{1}{2 \pi i} \int_{C^{\prime}} \frac{F_{n}(z)}{z} d z=\frac{1}{2 \pi} \int_{C^{\prime}} F_{n}(z) d \phi,
$$

for on $C^{\prime}$ it is true that $z=\rho e^{i \phi}, d z=i \rho e^{i \phi} d \phi=i z d \phi$. If in this formula we take the limit as a sequence of values $\rho$ approaches unity, the function $F_{n}(z)$ on $C^{\prime}$ approaches the limit $F_{n}(z)$ on $C$ almost everywhere (i.e. for all values of $\phi$ except at most a set of measure zero), and the functions $F_{n}\left(\rho e^{i \phi}\right)$ are uniformly bounded with respect to $\rho$ and $\phi$ for each value of $n$. It follows from a theorem due to Lebesgue that we may integrate term by term:

$$
1=\frac{1}{2 \pi} \int_{C} F_{n}(z) d \phi .
$$

Our theorem is now intuitively obvious. The values $F_{n}(z)$ can be plotted in the complex plane. If the values are suitably weighted, their average is unity, for the integral taken over $C$ can be interpreted as an average. This 
average lies on or within the smallest convex polygon which contains all the points $F_{n}(z)$, and lies on the polygon itself only in case the polygon degenerates. If $\lim _{n \rightarrow \infty}\left[\max \left|F_{n}(z)\right|\right]=1$, and if the average of the points $F_{n}(z)$ is always unity, then the values of $F_{n}(z)$ itself can differ but little from unity; if $n$ is sufficiently large this difference must be small except on a point set of small measure. In considering the average, values $F_{n}(z)$ which are taken on only on a set of points of zero measure may be neglected, and the theorem does not regard such values in the conclusion.

The intuitive proof just given can be expressed in more rigorous terms. We show that if an arbitrary $\eta>0$ be given, then $N$ exists such that

$$
\left|F_{n}(z)-1\right| \leqq \eta \text {, for } n \geqq N,
$$

except perhaps for $z$ on a point set of $C$ of measure less than or equal to $\eta$. If this statement is not exact, then there exists a positive $\eta$ such that

$$
\left|F_{n}(z)-1\right|>\eta
$$

for $z$ on a point set $E_{n}$ of $C$ of measure greater than $\eta$ and for an infinity of subscripts $n$. We use our original notation $F_{n}(z)$ for the functions of this subsequence and shall reach a contradiction. Choose $\epsilon$ so small that $\left|F_{n}(z)\right|<1+\epsilon$ together with $\left|F_{n}(z)-1\right|>\eta$ implies

$$
\Re\left[F_{n}(z)\right]<\eta^{\prime}<1 \text {, or } \Re\left[1-F_{n}(z)\right]>\eta^{\prime},
$$

for some $\eta^{\prime}>0$, where the symbol $\Re$ denotes the real part of the quantity which follows it. Such an $\epsilon$ exists, as is obvious geometrically; it is sufficient to choose $\epsilon<\left(1+\eta^{2}\right)^{1 / 2}-1$. Choose $\epsilon$ so small also that we have

On the one hand it follows that

$$
\epsilon<\frac{\eta \eta^{\prime}}{2 \pi-\eta} .
$$

$$
\frac{1}{2 \pi} \int_{C}\left[1-F_{n}(z)\right] d \phi=0,
$$

and on the other hand that

$$
\begin{aligned}
\Re\left\{\frac{1}{2 \pi} \int_{C}\left[1-F_{n}(z)\right] d \phi\right\} \\
=\frac{1}{2 \pi} \int_{E_{n}} \Re\left[1-F_{n}(z)\right] d \phi+\frac{1}{2 \pi} \int_{C\left(E_{n}\right)} \Re\left[1-F_{n}(z)\right] d \phi \\
\geqq \frac{\eta \eta^{\prime}}{2 \pi}-\frac{\epsilon(2 \pi-\eta)}{2 \pi}>0,
\end{aligned}
$$


which is a contradiction. In the proof, $C\left(E_{n}\right)$ denotes the set complementary to $E_{n}$, whose measure is not greater than $2 \pi-\eta$, and we have used the fact, obvious geometrically, that if $\left|F_{n}(z)\right|<1+\epsilon$, then $\Re\left[1-F_{n}(z)\right]>-\epsilon$.

Our preliminary theorem is now established, and leads to another preliminary theorem:

Let the single auxiliary condition of Theorem 2 be $F_{n}(0)=1$. Then we have on the circumference $C$

$$
\lim _{n \rightarrow \infty} \int_{0}^{2 \pi}\left|F_{n}(z)-1\right| d \phi=0 .
$$

Indeed we can easily establish the following:

If the uniformly bounded sequence of measurable functions $\left\{F_{n}(z)\right\}$ quasiconverges to the function $F(z)$ on the point set $E$ of finite measure, then we have

$$
\lim _{n \rightarrow \infty} \int_{E}\left|F_{n}(z)-F(z)\right|^{p}|d z|=0, p>0 .
$$

Assume $\left|F_{n}(z)\right| \leqq M$, and let $m$ be the measure of $E$. Then if an arbitrary positive $\epsilon$ be given, there exists $N$ so that we have, for $n \geqq N$,

$$
\left|F_{n}(z)-F(z)\right|<\epsilon
$$

except perhaps on a point set $E_{n}$ of measure less than $\epsilon$. Let $C\left(E_{n}\right)$ denote the set complementary (with respect to $E$ ) to $E_{n}$. There follows

$$
\begin{aligned}
\int_{E}\left|F_{n}(z)-F(z)\right|^{p}|d z|= & \int_{C\left(E_{n}\right)}\left|F_{n}(z)-F(z)\right|^{p}|d z| \\
& +\int_{E_{n}}\left|F_{n}(z)-F(z)\right|^{p}|d z|<m \epsilon^{p}+2^{p} M^{p} \epsilon
\end{aligned}
$$

which approaches zero with $\epsilon$, and the theorem is proved.

It is interesting to notice that the single condition

$$
\lim _{n \rightarrow \infty} \int_{0}^{2 \pi}\left|F_{n}(z)-1\right|^{p} d \phi=0, \quad p>0,
$$

for $z$ on $C$ implies the result, in the sense of quasiconvergence on $C$,

$$
\mathrm{q}_{n \rightarrow \infty} F_{n}(z)=1 \text {. }
$$

In fact, the corresponding condition in the general case

$$
\lim _{n \rightarrow \infty} \int_{C}\left|F_{n}(z)-F(z)\right|^{p} d \phi=0, \quad p>0,
$$


implies, in the sense of quasiconvergence on $C$,

$$
\operatorname{qlim}_{n \rightarrow \infty} F_{n}(z)=F(z) .
$$

Otherwise we should have, for some $\epsilon>0$ and for an infinity of indices $n$,

$$
\left|F_{n}(z)-F(z)\right| \geqq \epsilon
$$

on a point set of measure greater than or equal to $\epsilon$, and hence for those indices

$$
\int_{C}\left|F_{n}(z)-F(z)\right|^{p} d \phi>\epsilon^{p+1},
$$

which is impossible.

The results we have now established lead directly to the following:

In Theorems 2 and 3 we have, in the sense of quasiconvergence on the circumference $C$,

$$
\underset{n \rightarrow \infty}{\mathrm{q}} \lim _{n}(z)=F(z), \quad \operatorname{qlim}_{n \rightarrow \infty} f_{n}(z)=f(z) .
$$

We have also

$$
\lim _{n \rightarrow \infty} \int_{C}\left|F_{n}(z)-F(z)\right|^{p} d \phi=0, \quad \lim _{n \rightarrow \infty} \int_{C}\left|f_{n}(z)-f(z)\right|^{p} d \phi=0, \quad p>0 .
$$

Transform the functions $F(z)$ and $F_{n}(z)$ as in $\S 5$, using formulas (5.3), (5.4), (5.10), (5.11), repeated as often as necessary and in the proper order. In the last stage we have a situation of type

$$
\lim _{n \rightarrow \infty} \phi_{n i}(z)=\phi_{i}(z),|z|<1,
$$

where $\phi_{i}(z)$ is identically a constant $\phi_{i}$ of modulus $M$, and where the auxiliary conditions to which the $\phi_{n i}(z)$ are subjected are of the form $\phi_{n i}\left(z^{\prime}\right)=\phi_{i}$, where $z^{\prime}$ is a particular point interior to $C$. At this stage the theorem is true, as is obvious by considering the functions $\phi_{n i}(z) / \phi_{i}$, and by transforming with the substitution $w=\left(z-z^{\prime}\right) /\left(1-\bar{z}^{\prime} z\right)$. For these transformed functions, the theorem reduces to the special case already considered. Hence by transforming again by the inverses of the previous transformations, in the inverse order, we see that the result stated is valid for $F(z)$ and $F_{n}(z)$ and therefore for $f(z)$ and $f_{n}(z)$.

It is conceivable that there should be difficulty in this application, due to the fact that the normal to $C$ does not remain the normal to $C$ under transformations of the form $w=\left(z-z^{\prime}\right) /\left(1-\bar{z}^{\prime} z\right)$. This difficulty is apparent rather than actual, however, for Fatou's theorem applies to approach to $C$ not 
merely along the normal to $C$ but also in any way in a triangle interior (except for a single vertex) to $C$. $^{*}$

The result on quasiconvergence just proved can, by conformal mapping, be extended to an arbitrary simply connected region $B$ in the situation of Theorem 14, provided that the boundary values of the functions $\psi_{n}(w)$ are suitably defined-let us say by normal approach to the boundary after $B$ is mapped onto a circle - and provided that the term measure on $C$ is also properly interpreted, perhaps by measure on the unit circle when $B$ is mapped onto its interior. If $B$ is the interior of a rectifiable Jordan curve, however, the boundary values can be taken by approach along the normal to $C$, and the definition of measure can be taken in terms of arc length on $C$. For a point set of zero measure on the curve $C$ corresponds to a point set of zero measure on the unit circle, and conversely. $\dagger$ Moreover, approach almost everywhere to the boundary $C$ of $B$ in a triangle in $B$ means approach almost everywhere to the boundary of the unit circle in a triangle in that unit circle, and reciprocally, for if $B$ is mapped onto the interior of the unit circle, the mapping is conformal almost everywhere on the boundary. $\ddagger$ The result

$$
\lim _{n \rightarrow \infty} \int_{C}\left|F_{n}(z)-F(z)\right|^{p} d s=0, \quad p>0,
$$

also naturally persists under the present circumstances, that $B$ is bounded by a rectifiable Jordan curve $C$.

In the numerous cases we have considered of approximation by Tchebycheff polynomials on the boundary $C$ of a region $B$ in the w-plane, the question of boundary values of the approximating functions $F_{n}(z)$ is automatically answered. For the Tchebycheff polynomials are continuous on and within the boundary $C$, and under conformal mapping approach to the unit circle $\gamma$ in the $z$-plane corresponds to approach to the boundary $C$ in the $w$-plane. If $z$ approaches along the radius a point of $\gamma$ so that the corresponding point $w$ approaches an accessible point of $C$, then a unique limit for the corresponding functional values of the approximating functions obviously exists in the

* It is not intended to imply, nor is it essential to our argument, that whenever the limit for radial approach exists, then the limit in this new sense also exists. It is sufficient for our purpose that the limit in this new sense exists almost everywhere.

† This theorem is due to Lusin. See Lusin and Privaloff, Annales de l'École Normale Supérieure, (3), vol. 42 (1925), p. 156.

‡ Carathéodory, Schwarz Festschrift, Berlin, 1914, pp. 19-41; pp. 40-41. It is there shown that the existence of a tangent implies conformality on the boundary, but for a rectifiable curve a tangent is known to exist almost everywhere. The method of proof used here gives a proof of Denjoy's extension of Fatou's theorem, to regions $B$ bounded by arbitrary rectifiable Jordan curves. Compare Denjoy, Paris Comptes Rendus, vol. 168 (1919), p. 387. 
$w$-plane and hence also in the z-plane. By Fatou's theorem, $w$ can fail to approach a limit when $z$ approaches $\gamma$ along a radius at most on a set of measure zero on $\gamma$, so almost everywhere on $C$ the actual boundary values of the Tchebycheff polynomials are the boundary values in the sense of Fatou in the z-plane, provided "almost everywhere" is interpreted in terms of measure on the unit circle $\gamma$ in the $z$-plane.

It is interesting to note that the result we have proved for quasiconvergence on the boundary $C$ implies convergence in the interior of $B$. We prove the general result in the $z$-plane, which implies the result also in the w-plane.

If the sequence of functions $\left\{F_{n}(z)\right\}$ each analytic and bounded interior to $C:|z|=1$ is uniformly bounded almost everywhere on $C$ (that is, the sequence of boundary values taken on almost everywhere is uniformly bounded) and approaches on $C$ in the sense of quasiconvergence the function $F(z)$, the boundary values* (taken on almost everywhere on $C$ ) of a function $F(z)$ analytic and bounded interior to $C$, then we have

$$
\lim _{n \rightarrow \infty} F_{n}(z)=F(z)
$$

throughout the interior of $C$, uniformly on any closed point set interior to $C$.

In this theorem, boundary value means naturally boundary value in the sense of Fatou.

We have by the boundedness of the individual functions $F_{n}(z)$ and $F(z)$ as above, by integrating first over the circle $C^{\prime}:|z|=\rho<1$ and then allowing a sequence of values $\rho$ to approach unity,

$$
F_{n}(z)-F(z)=\frac{1}{2 \pi i} \int_{C} \frac{F_{n}(t)-F(t)}{t-z} d t .
$$

The function $1 /(t-z)$ is uniformly bounded in $t$ and $z$ if we have $|z| \leqq \rho<1$ :

$$
\left|\frac{1}{2 \pi i} \int_{C} \frac{F_{n}(t)-F(t)}{t-z} d t\right| \leqq \frac{1}{2 \pi i(1-\rho)} \int_{C}\left|F_{n}(t)-F(t)\right| d \phi ;
$$

the last integral approaches zero, so we have

$$
\lim _{n \rightarrow \infty} F_{n}(z)=F(z)
$$

interior to $C$, uniformly on any closed point set interior to $C$.

* The equation

$$
\mathrm{q}_{n \rightarrow \infty} F_{n}(z)=F(z)
$$

for $z$ on $C$ under the present hypothesis on the sequence $\left\{F_{n}(z)\right\}$ implies the fact that $F(z)$ is the boundary values of a function analytic and bounded interior to $C$. 
In this same order of ideas we can prove the following:

Let $f(z)$ be analytic and bounded for $|z|<1$ :

$$
|f(z)| \leqq N \text {. }
$$

If we have almost everywhere (i.e. for almost all values of $\phi$ )

then we have

$$
\overline{\operatorname{bound}_{\rho \rightarrow 1}} f\left(\rho e^{i \phi}\right) \leqq M, \phi \text { constant }
$$

$$
|f(z)| \leqq M \text { for }|z|<1 .
$$

We have Cauchy's integral

$$
f(0)=\frac{1}{2 \pi i} \int_{C^{\prime}} \frac{f(z) d z}{z}=\frac{1}{2 \pi} \int_{C^{\prime}} f(z) d \phi,
$$

where $C^{\prime}$ is the circle $|z|=\rho<1$. If we take the limit as $\rho$ approaches unity, we have

$$
f(0)=\frac{1}{2 \pi} \int_{C} f(z) d \phi,
$$

where this integral refers as usual to the boundary values of $f(z)$, namely $\lim _{\rho \rightarrow 1} f\left(\rho e^{i \phi}\right)$; this limit is almost everywhere not greater than $M$ in absolute value, so we have

$$
|f(0)| \leqq M
$$

Let $z_{0}$ be any other point interior to $C$. We transform by setting $w=\left(z-z_{0}\right)$ $\cdot\left[1-\bar{z}_{0} z\right]^{-1}$, so that $C$ is transformed into itself and $z_{0}$ into $w=0$. By Fatou's theorem, the boundary values of $f(z)$ in the w-plane for radial approach to $C$ are equal almost everywhere to the boundary values of $f(z)$ in the $z$-plane for radial approach to $C$. Then we have in the w-plane almost everywhere on $|w|=1$, for the boundary values obtained by radial approach, $|f(z)| \leqq M$, from which it follows that the functional value for $w=0$ is also not greater than $M$ in absolute value:

$$
\left|f\left(z_{0}\right)\right| \leqq M
$$

and the proof is complete.

12. Miscellaneous problems. We shall consider in this section various problems, some solved and others unsolved, and make a few additional remarks relative to the problems already treated in detail.

12.1. As a first problem, we compare the two measures of approximation, least squares and that of Tchebycheff. For the sake of simplicity* we restrict

* The results on approximation by least squares hold with some changes also for much more general curves and functions; see Szegö, Mathematische Zeitschrift, vol. 9 (1921), pp. 218-270. 
ourselves to the case of approximation on the unit circle $C$ to a rational function $r(z)$ with no singularities on $C$.

The least-squares method of approximation to $r(z)$ on $C$ by polynomials in $z$ is to study the sequence of polynomials $p_{n}(z)$ of respective degrees $n$ which make the integral

$$
\int_{C}\left|r(z)-p_{n}(z)\right|^{2} d s
$$

a minimum. If we set $r(z)=r_{1}(z)+r_{2}(z)$; where $r_{1}(z)$ is analytic on and within $C$ and where $r_{2}(z)$ is analytic on and exterior to $C$ and vanishes at infinity, then the approximating polynomials $p_{n}(z)$ for approximation to $r(z)$ are the successive convergents of the Taylor expansion of $r_{1}(z)$ :

$$
\begin{aligned}
& r_{1}(z)=a_{0}+a_{1} z+a_{2} z^{2}+\cdots, \\
& p_{n}(z)=a_{0}+a_{1} z+a_{2} z^{2}+\cdots+a_{n} z^{n}
\end{aligned}
$$

this determination of $p_{n}(z)$ follows from the formulas for the coefficients of $p_{n}(z)$ :

$$
a_{n}=\frac{1}{2 \pi} \int_{C} r(z) \bar{z}^{n} d s=\frac{1}{2 \pi i} \int_{C} r(z) \frac{d z}{z^{n+1}} .
$$

If we approximate $r(z)$ on $C$ by means of polynomials $p_{-n}(z)$ in $1 / z$ which vanish at infinity, then the method of least squares yields as the polynomials $p_{-n}(z)$ the successive convergents of the Taylor expansion at infinity of $r_{2}(z)$ :

$$
\begin{aligned}
r_{2}(z) & =a_{-1} z^{-1}+a_{-2} z^{-2}+\cdots, \\
p_{-n}(z) & =a_{-1} z^{-1}+a_{-2} z^{-2}+\cdots+a_{-n} z^{-n} .
\end{aligned}
$$

Thus we have the simple formulas $\lim _{n \rightarrow \infty} p_{n}(z)=r_{1}(z)$, for $|z|$ less than some $R>1$, uniformly for $|z| \leqq R_{1}<R ; \lim _{n \rightarrow \infty} p_{-n}(z)=r_{2}(z)$, for $|z|$ greater than some $R^{\prime}<1$, uniformly for $|z| \geqq R_{1}^{\prime}>R^{\prime}$. There results from these two equations

$$
r(z)=\lim _{n \rightarrow \infty} p_{n}(z)+\lim _{n \rightarrow \infty} p_{-n}(z),
$$

uniformly for $z$ on $C$.

The circumstances are not nearly so simple for approximation by the method of Tchebycheff. Let $\left\{\tau_{n}(z)\right\}$ be the sequence of polynomials in $z$ for approximation on $C$ to $r(z)$, and $\left\{\tau_{-n}(z)\right\}$ the sequence of polynomials in $1 / z$ for approximation on $C$ to $r(z)$, subject to the restriction* $\tau_{-n}(\infty)=0$. We

* This restriction is not essential either here or above, but is a matter of convenience here, not merely to afford an analogy with the method of least squares, but to avoid complication in the present formulas. If the restriction is not made, the number $a_{0}$ enters in an extraneous manner. 
now have, if $f(z)$ denotes the minimizing function of Theorem 3 for $r_{2}(z)$, $\lim _{n \rightarrow \infty} \tau_{n}(z)=f(z)+r_{1}(z)$, for $|z|$ less than some $R>1$, uniformly for $|z|$ $\leqq R_{1}<R ; \lim _{n \rightarrow \infty} \tau_{-n}(z)=f_{1}(z)+r_{2}(z)$, for $|z|$ greater than some $R^{\prime}<1$, uniformly for $|z| \geqq R_{1}^{\prime}>R^{\prime}$; here the function $f_{1}(z)$ corresponds to approximation to $r_{1}(z)$ on $C$ by functions analytic exterior to $C$, with the auxiliary condition $f_{1}(\infty)=0$. Each of these two limits depends on both $r_{1}(z)$ and $r_{2}(z)$-of course $r_{1}(z)$ and $r_{2}(z)$ can be chosen independently of each other-and hence, in contrast to the limits with the method of least squares, there is no simple obvious relation connecting with $r(z)$ the sum of the two limits.

It would be interesting to study approximation in the sense of least squares of an arbitrary function (continuous or not) $f(z)$ defined on a rectifiable Jordan curve, to determine as we have done for simple functions in the corresponding case of Tchebycheff approximation, the properties of the sequences $\left\{p_{n}(z)\right\},\left\{p_{-n}(z)\right\}$ defined as above.* It will be noticed that for the circle $C$ and for a function $f(z)$ analytic on $C$, the sequence $\left\{p_{n}(z)\right\}$ has the limit

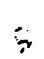

$$
\frac{1}{2 \pi i} \int_{C} \frac{f(t) d t}{t-z} .
$$

In the more general case, too, there is intimate connection with Cauchy's integral and with Fourier's series. The present writer hopes soon to publish some results on this general topic.

12.2. We consider now some of the cases of approximation already treated, modified so that the auxiliary conditions refer to points some of which may be outside of the point set on which the given function is approximated. To be sure, we have ordinarily considered approximation on the boundary $C$ of a region, and auxiliary conditions not on $C$ but interior to $C$, but if the functions involved have no singularities interior to $C$, this is not essentially different from approximation in the corresponding closed region with the same auxiliary conditions. In the present case the auxiliary conditions refer to points entirely without restriction as to location. The simplest situation is a generalization of Theorem 4:

Let the function $f(z)$ be analytic on the closed limited point set $C$, not a single point, whose complementary set with respect to the entire plane is simply connected. Let $\left\{\pi_{n}(z)\right\}$ be the sequence of Tchebycheff polynomials for approximation to $f(z)$ on $C$ with the auxiliary conditions

* Szegö (loc. cit., p. 239, footnote) also contrasts the two measures of approximation, least squares and that of Tchebycheff, but for less general cases: (1) where the given function is analytic in the closed region considered, (2) where the given function is $z^{n}$ and the approximating polynomial of degree $n-1$. 
$\pi_{n}\left(\alpha_{i}\right)=f\left(\alpha_{i}\right), \alpha_{i}$ on $C$

$(i=1,2, \cdots, k)$,

$\pi_{n}\left(\beta_{i}\right)=\gamma_{i}, \beta_{i}$ not on $C$

$\left(i=1,2, \cdots, k^{\prime}\right)$.

Let $C_{R}$ denote the largest curve of the family $C_{\rho}$ which contains within it no singular point of $f(z)$ and no point $\beta_{i}$ at which the assigned value $\gamma_{i}$ differs from the value $f\left(\beta_{i}\right)$ of the analytic extension of $f(z)$. Then the sequence $\left\{\pi_{n}(z)\right\}$ converges everywhere interior to $C_{R}$, uniformly on any closed point set interior to $C_{R}$; interior to $C_{R}$ the limit of the sequence is naturally the function $f(z)$ or an analytic extension of that function.

In this theorem we require the suitable derivatives of $\pi_{n}(z)$ to coincide with those of $f(z)$, in case not all of the points $\alpha_{i}$ are distinct, and we also require the suitable derivatives $\pi_{n}^{(j)}\left(\beta_{i}\right)$ to coincide with the derivatives of $f^{(j)}\left(\beta_{i}\right)$ at multiple points $\beta_{i}$ interior to $C_{R}$.

Let us introduce the notation

$$
\pi(z)=\left(z-\alpha_{1}\right) \cdots\left(z-\alpha_{k}\right)\left(z-\beta_{1}\right) \cdots\left(z-\beta_{k^{\prime}}\right),
$$

and let $p(z)$ be a polynomial of degree $k+k^{\prime}-1$ such that

$$
\begin{array}{ll}
p\left(\alpha_{i}\right)=f\left(\alpha_{i}\right) & (i=1,2, \cdots, k), \\
p\left(\beta_{i}\right)=\gamma_{i} & \left(i=1,2, \cdots, k^{\prime}\right) .
\end{array}
$$

The function

$$
F(z)=\frac{f(z)-p(z)}{\pi(z)}
$$

is analytic everywhere within $C_{R}$, so by Theorem 4 there exist polynomials $P_{n}(z)$ of respective degrees $n$ such that

$$
\left|F(z)-P_{n}(z)\right| \leqq \frac{M_{1}}{R_{1}^{n}}, \text { for } z \text { on } C, R_{1}<R .
$$

It follows, since $\pi(z)$ is uniformly bounded on $C$, that we have

$$
\left|[f(z)-p(z)]-\pi(z) P_{n}(z)\right| \leqq \frac{M_{2}}{R_{1}^{n}}, \text { for } z \text { on } C,
$$

which we write in the form

$$
\left|f(z)-\left[p(z)+\pi(z) P_{n}(z)\right]\right| \leqq \frac{M_{2}}{R_{1}^{n}}, \text { for } z \text { on } C .
$$

The quantity in square brackets can be considered a polynomial of degree which we indicate by $m=n+k+k^{\prime}$, so the inequality can be written 
(12.1) $\left|f(z)-p_{m}(z)\right| \leqq \frac{M_{3}}{R_{1}{ }^{m}}$, for $z$ on $C$, where $p_{m}(z)=p(z)+\pi(z) P_{n}(z)$.

To be sure, this inequality and the remark on the degree of $p_{m}(z)$ have been established not for all $m$ but merely for $m$ sufficiently large, but the introduction of suitable polynomials for the lacking values of $m$ presents no difficulty.

We have exhibited a set of polynomials which satisfy inequality (12.1) and for $m$ sufficiently large satisfy the prescribed auxiliary conditions; the Tchebycheff polynomials must therefore likewise satisfy this inequality for $m$ sufficiently large, so that the theorem follows from Theorem 4 .

It will be noted that the sequence $\left\{\pi_{n}(z)\right\}$ can converge uniformly in no region $C_{R_{1}}$, with $R_{1}>R$.

It is clear from the reasoning just given what modifications are to be made in Theorems 10,13,15, if auxiliary conditions for points exterior to $C$ are introduced; we leave the results to the reader. If auxiliary conditions for points exterior to $C$ are introduced in these theorems, there are of course no modifications in the conclusions of the theorems to be made so far as concerns the general regions $B$ not assumed bounded by analytic curves.*

12.3. Another problem related to those discussed in detail is that of approximation by functions other than rational polynomials. We take up the situation of Theorem 13 by way of illustration. Let $\left\{\chi_{n}(w)\right\}$ be a set of functions continuous on $C$; compare the latter part of $\$ 6$. Let $F(w)$ be an arbitrary function continuous on $C$. The function $\Sigma_{n}(w)$ of the form

$$
\Sigma_{n}^{\prime}(w)=a_{1} \chi_{1}(w)+\cdots+a_{n} \chi_{n}(w)
$$

such that

$$
\max \left|\Sigma_{n}^{\prime}(w)-F(w)\right|, w \text { on } C,
$$

has the least possible value, $n$ being fixed, may be called the Tchebycheff polynomial $\Sigma_{n}(w)$ of order $n$ for approximation to $F(w)$ on $C$. When the functions $\Sigma_{n}(w)$ exist-this need not be the case-the questions naturally arise: What is

$$
\lim _{n \rightarrow \infty}\left[\max \left|\Sigma_{n}(w)-F(w)\right|, w \text { on } C\right] ?
$$

What can be said of the convergence of the sequence $\left\{\Sigma_{n}(w)\right\}$ on $C$ and interior to $C$ ? What is the limit of this sequence?

Whenever the set $\left\{\chi_{n}(w)\right\}$ forms a basis for $B$, that is, if the functions $\chi_{n}(w)$ are analytic in $B$, continuous on $B+C$, and if an arbitrary function

* Compare Walsh, these Transactions, vol. 31 (1929), pp. 477-502, \$7. This illustrates other restrictions on the sequence $\left\{\pi_{n}(z)\right\}$. 
analytic on and within $C$ can be uniformly approximated on $B+C$ as closely as desired by a function of type (12.2), and if each function $\chi_{n}(w)$ can be so approximated on $B+C$ by a rational polynomial, then for any function $F(w)$ the limit (12.3) is the same for the functions $\Sigma_{n}(w)$ as for the rational Tchebycheff polynomials. In particular if $F(w)$ is the function $s(w)$ of Theorem 13, this limit is $M$ and we have

$$
\lim _{n \rightarrow \infty} \Sigma_{n}(w)=g(w),
$$

uniformly on any closed point set interior to $B$. This result obviously requires the existence of the Tchebycheff polynomial (12.2), but does not require its uniqueness. For special sets of functions $\left\{\chi_{n}(w)\right\}$, more explicit results are obtainable.

The study of a basis for a given region should be interesting also in the case that the given region is not simply connected. So far as the writer is aware, even the analogue of Theorem 1 has not been established in this case, although most of the reasoning for simply connected regions, with only minor modifications, would seem to carry over.

12.4. We mention a few other problems suggested by and related to the present discussion. Most obvious is perhaps the study of the rational Tchebycheff polynomial with auxiliary conditions, for approximation to a real function $f(x)$ on an interval or other linear point set $C$, where the auxiliary conditions refer to points of $C$ and also to points not belonging to $C$, where $f(x)$ is or is not continuous on $C$, and when the auxiliary conditions on $C$ do or do not coincide with the values of $f(x)$. Much of the discussion already given in the present paper applies to real polynomials, and many of the classical properties of the Tchebycheff polynomial can be readily extended to the new case.*

The present problems (e.g. of Theorem 10) should also be interesting if $C$ is a Jordan arc or more general point set, particularly where the given points $P$ lie on $C$. What can be said of the sequence of functions corresponding to the regions $C_{\mu}$ ? What occurs in Theorem 15 if some of the points $\alpha_{i}$ lie on $C$ itself?

Approximation is ordinarily considered, moreover, in the sense of addition or subtraction, as in the present paper, but related questions are easily framed. Let $f(z)$ be a given rational function of $z$. What function $\phi(z)$, perhaps satisfying certain auxiliary conditions, is such that

$$
\overline{\text { bound }}|\phi(z) f(z)|
$$

* Compare de la Vallée Poussin, loc. cit. In particular the proof of the existence of the Tchebycheff polynomial does not require the continuity of $f(x)$. 
for $|z| \rightarrow 1$ is least? is such that this upper bound for $|z| \leqq 1$ is least? Does a polynomial $\phi_{n}(z)$ of given degree $n$ exist which satisfies the auxiliary conditions and for which this upper bound is unique? Is it true that $\lim _{n \rightarrow \infty}$ $\phi_{n}(z)=\phi(z)$ uniformly for $|z| \leqq 1$ ? Some of the methods of the present paper serve to answer these questions and others, at least in part, and the writer hopes to consider these problems on another occasion.

HARVARD UNIVERSITY,

Caubridge, Mass. 